\title{
Reflected BSDEs, optimal control and stopping for infinite-dimensional systems
}

\author{
Marco Fuhrman \\ Politecnico di Milano, Dipartimento di Matematica \\ via Bonardi 9, 20133 Milano, Italy \\ e-mail: marco.fuhrman@polimi.it \\ Federica Masiero, Gianmario Tessitore \\ Dipartimento di Matematica e Applicazioni, Università di Milano Bicocca \\ via Cozzi 55, 20125 Milano, Italy \\ e-mail: federica.masiero@unimib.it, gianmario.tessitore@unimib.it
}

\begin{abstract}
We introduce the notion of mild supersolution for an obstacle problem in an infinite dimensional Hilbert space. The minimal supersolution of this problem is given in terms of a reflected BSDEs in an infinite dimensional Markovian framework. The results are applied to an optimal control and stopping problem.
\end{abstract}

\section{Introduction}

The connection between backward stochastic differential equations (BSDEs) in $\mathbb{R}^{n}$ and semilinear parabolic PDEs is known since the seminal paper of Pardoux and Peng [18]. This result was extended to the case of reflected BSDEs and of obstacle problem for PDEs in [6]. Moreover it is also well known that BSDEs are related to optimal stochastic control problems and that reflected BSDEs are related to optimal stopping or optimal control/stopping problems, see [19]. We notice that in the finite dimensional framework the above mentioned partial differential equations are intended either in classical sense (see [18]) or, more frequently, in viscosity sense.

On the other hand the relation among backward stochastic differential equations in infinite dimensional spaces, optimal control of stochastic evolution equations with values in Hilbert spaces and parabolic equations in infinite dimensional spaces was investigated in [10] and in several successive papers. In the above mentioned literature it appears that the concept of solution of the PDE has to be modified in the infinite dimensional case. Namely classical solutions require too much regularity while the theory of viscosity solutions can be applied only in special cases with trace class noise and very regular value function (see [9]). The type of definition that was seen to fit the infinite dimensional framework and the BSDE approach is the classical notion of mild solution. Namely if, on an Hilbert space $H$, we consider a semilinear parabolic PDE such as

$$
\left\{\begin{array}{l}
\frac{\partial u}{\partial t}(t, x)=\mathcal{L}_{t} u(t, x)+\psi(t, x, u(t, x), \nabla u(t, x)) \\
u(T, x)=\phi(x),
\end{array} \quad t \in[0, T], x \in H\right.
$$

and $\left(P_{s, t}\right)_{0<s<t<T}$ is the transition semigroup related to the second order differential operators $\left(\mathcal{L}_{t}\right)_{t \in[0, T]}$ then a function $u:[0, T] \times H \rightarrow \mathbb{R}$ is called a mild solution of the above PDE whenever 
$u$ admits a gradient (in a suitable sense) and it holds:

$$
u(s, x)=P_{s, T}[\phi](x)+\int_{s}^{T} P_{s, \tau}[\psi(\tau, \cdot, u(\tau, \cdot), \nabla u(\tau, \cdot))](x) d \tau .
$$

Large amount of literature has then extended the BSDE approach to control problems to several different situations both in the finite and in the infinite framework but, at our best knowledge, the problem of relating reflected BSDEs in infinite dimensional spaces and obstacle problems for PDEs with infinitely many variables was never investigated. The point is that it is not obvious how one should include the reflection term (which is not absolutely continuous with respect to Lebesgue measure on $[0, T]$ ) into the definition of mild solution.

In this paper, to overcome such a difficulty and inspired by the techniques in A. Bensoussan [2], we propose the notion of mild-supersolution. To be more specific, our main result (see Theorem 3.3) will be to prove that if $\left(X^{s, x}, Y^{s, x}, Z^{s, x}, K^{s, x}\right)$ is the solution of the following forward backward system with reflected BSDE:

$$
\left\{\begin{array}{l}
d X_{t}^{s, x}=\left(A X_{t}^{s, x}+F\left(t, X_{t}^{s, x}\right)\right) d t+G\left(t, X_{t}^{s, x}\right) d W_{t} \quad t \in[s, T] \\
X_{s}^{s, x}=x \\
-d Y_{t}^{s, x}=\psi\left(t, X_{t}^{s, x}, Y_{t}^{s, x}, Z_{t}^{s, x}\right) d t+d K_{t}^{s, x}-Z_{t}^{s, x} d W_{t}, \quad t \in[s, T], \\
Y_{T}^{s, x}=\phi\left(X_{T}^{s, x}\right) \\
Y_{t}^{s, x} \geq h\left(X_{t}^{s, x}\right), \\
\int_{s}^{T}\left(Y_{t}^{s, x}-h\left(X_{t}^{s, x}\right)\right) d K_{t}^{s, x}=0 .
\end{array}\right.
$$

setting $u(t, x):=Y_{t}^{t, x}$ then $u$ is the minimal mild supersolution of the obstacle problem

$$
\begin{cases}\min \left(u(t, x)-h(x),-\frac{\partial u}{\partial t}(t, x)-\mathcal{L}_{t} u(t, x)-\psi(t, x, u(t, x),\right. & \nabla u(t, x) G(t, x)))=0 \\ u(T, x)=\phi(x), & t \in[0, T], x \in H\end{cases}
$$

see Definition 3.2 where the notion of supersolution is introduced. As we explain with some more details in Remark 3.2, the notion of supersolution that we propose here is not related to viscosity theory and is inspired by the work of A. Bensoussan, see [2]. Here we are able to characterize the value function as the minimal mild supersolution of the corresponding HJB obstacle problem. This seems natural in our context since it corresponds to the minimality requirement included in the definition of solution to reflected BSDE (see [6]) and somehow bypasses the question of uniqueness of the solution to the obstacle problem. A more explicit notion of uniqueness remains an open issue.

Another issue of this paper is that we do not assume any nondegeneracy on the coefficient $G$ (and consequently any strong ellipticity on the second order differential operator in the PDE). Therefore (see the Example at the beginning of Section 3.1) we can not expect to have regular solutions of the obstacle problem nor regular value functions of the optimal stopping problem. In particular the directional gradient $\nabla u G$ can not be understood according to its classical formulation. We employ the definition of generalized gradient (in probabilistic sense) introduced in [13]. It was proved in [13] that such generalized gradient exists for all locally Lipschitz functions. In Theorem 2.10 we prove that our candidate solution $u(t, x):=Y_{t}^{t, x}$ is indeed locally Lipschitz. Moreover we notice that we work under general growth assumptions with respect to $x$ on the nonlinear term $\psi$ and on the final datum $\phi$. This forces us to obtain $L^{p}$ estimates on the solution of the reflected BSDE that extend the ones proved in [6].

The structure of the paper is the following. In section 2 we study reflected BSDEs obtaining the desired $L^{p}$ estimates and the local Lipschitzianity with respect to the initial datum in the 
markovian framework. In section 3 we introduce the notion of minimal mild supersolution of the obstacle problem in the sense of the generalized gradient and we show how it is related to the reflected BSDEs. Finally in section 4 we apply the above results to an optimal control and stopping problem.

\section{Reflected BSDEs}

In a complete probability space $(\Omega, \mathcal{F}, \mathbb{P})$ we consider a cylindrical Wiener process $\left\{W_{t}, t \geq 0\right\}$ with values in an Hilbert space $\Xi$ and we denote by $\left(\mathcal{F}_{t}\right)_{t \geq 0}$ is its natural filtration, augmented in the usual way. We consider the following reflected backward stochastic differential equation (RBSDE in the following):

$$
\left\{\begin{array}{l}
d Y_{t}=-f\left(t, Y_{t}, Z_{t}\right) d t-d K_{t}+Z_{t} d W_{t}, \quad t \in[0, T] \\
Y_{T}=\xi \\
Y_{t} \geq S_{t} \\
\int_{0}^{T}\left(Y_{t}-S_{t}\right) d K_{t}=0
\end{array}\right.
$$

for the unknown adapted processes $Y, Z$ and $K$. In the above equation $Y$ and $K$ are real processes while $Z$ is a $\Xi^{*}$-valued process. Moreover $Y$ admits a continuous modification and $K$ is a continuous non-decreasing process with $K_{0}=0$. The equation is understood in the usual integral way, namely:

$$
Y_{t}+\int_{t}^{T} Z_{r} d W_{r}=\xi+\int_{t}^{T} f\left(r, Y_{r}, Z_{r}\right) d r+K_{T}-K_{t}, \quad t \in[0, T], \mathbb{P}-\text { a.s.. }
$$

In the following, if $E$ is a separable Hilbert space, $0<a<b$ and $p \geq 1$ by $L_{\mathcal{P}}^{p}(\Omega \times[a, b], E)$ we denote the space of $E$-vauled $\left(\mathcal{F}_{t}\right)$-predictable processes $\ell$ such that:

$$
\mathbb{E} \int_{a}^{b}|\ell(t)|^{p} d t<\infty
$$

If $E=\mathbb{R}$ we write $L_{\mathcal{P}}^{p}(\Omega \times[a, b])$ instead of $L_{\mathcal{P}}^{p}(\Omega \times[a, b], \mathbb{R})$.

Moreover $L_{\mathcal{P}}^{p}\left(\Omega, L^{2}([a, b], E)\right), p \geq 2$, we denote the subspace of $L_{\mathcal{P}}^{2}(\Omega \times[a, b], E)$ given by processes verifying

$$
\mathbb{E}\left[\int_{a}^{b}|\ell(t)|^{2} d t\right]^{p / 2}<\infty .
$$

and by $L_{\mathcal{P}}^{p}(\Omega, C([a, b], E))$ the subspace of $L_{\mathcal{P}}^{p}(\Omega \times[a, b], E)$ given by processes admitting a continuous version and verifying

$$
\mathbb{E} \sup _{t \in[a, b]}|\ell(t)|^{p}<\infty .
$$

An analogous definition is given to $L_{\mathcal{P}}^{p}\left(\Omega, L^{2}([a, b])\right)$ and to $L_{\mathcal{P}}^{p}(\Omega, C([a, b]))$

Finally by $\mathcal{P}$ we denote the predictable $\sigma$-algebra on $\Omega \times[0, T]$, and by $\mathcal{B}(\Lambda)$ the Borel $\sigma$-algebra on any topological space $\Lambda$

The next result was proven in [6] (see $\S 6$ and Lemma 6.1) and it is reported here for the reader's convenience. We omit the proof that remains the same. More precisely, while $Y$ and $K$ are real valued processes as in [6], here both $\left(W_{t}\right)_{t \geq 0}$ and $\left(Z_{t}\right)_{t \geq 0}$ take values in a general Hilbert space rather than in $\mathbb{R}^{d}$. However, all the arguments in [6] can be repeated just by replacing the norm of $\mathbb{R}^{d}$ by the natural Hilbertian norms. 
We introduce here the following class of penalized BSDEs:

$$
\left\{\begin{array}{l}
-d Y_{t}^{n}=f\left(t, Y_{t}^{n}, Z_{t}^{n}\right) d t+n\left(Y_{t}^{n}-S_{t}\right)^{-} d t-Z_{t}^{n, s, x} d W_{t}, \quad t \in[0, T] \\
Y_{T}^{n}=\xi
\end{array}\right.
$$

We denote by $\left(Y^{n}, Z^{n}\right) \in L_{\mathcal{P}}^{2}(\Omega, C([0, T])) \times L_{\mathcal{P}}^{2}(\Omega \times[0, T], \Xi)$, their unique mild solution (see [10]) and by $K^{n}$ the adapted, continuous, non-decreasing process: $K_{t}^{n}:=n \int_{0}^{t}\left(Y_{s}^{n}-S_{s}\right)^{-} d s$.

Theorem 2.1 Assume that $f: \Omega \times[0, T] \times \mathbb{R} \times \Xi \rightarrow \mathbb{R}$ is measurable with respect to $\mathcal{P} \otimes$ $\mathcal{B}\left(\mathbb{R} \times \Xi^{*}\right)$ and is Lipschitz with respect to $y$ and $z$ uniformly in $t$ and $\omega$. Moreover $f(\cdot, 0,0) \in$ $L_{\mathcal{P}}^{2}(\Omega \times[0, T])$ and $\xi \in L^{2}\left(\Omega, \mathcal{F}_{T}, \mathbb{P}\right)$. Finally assume that the obstacle $S$ is a continuous adapted real valued process satisfying

$$
\mathbb{E} \sup _{t \in[0, T]}\left|S_{t}\right|^{2}<\infty
$$

Then equation (2.1) admits a unique solution $(Y, Z, K)$ such that $Y \in L_{\mathcal{P}}^{2}(\Omega, C([0, T])), Z \in$ $L_{\mathcal{P}}^{2}\left(\Omega \times[0, T], \Xi^{*}\right)$ and $K$ is an adapted continuous non decreasing process with $K_{0}=0$ and $\mathbb{E}\left|K_{T}\right|^{2}<\infty$.

Moreover the following uniform estimate holds:

$$
\mathbb{E} \sup _{s \in[0, T]}\left|Y_{s}^{n}\right|^{2}+\mathbb{E} \int_{0}^{T}\left|Z_{t}^{n}\right|^{2} d t+c \mathbb{E}\left|K_{T}^{n}\right|^{2} \leq c \mathbb{E}|\xi|^{2}+c \mathbb{E} \int_{0}^{T}|f(t, 0,0)|^{2} d t,
$$

for a constant $c$ that does not depend on $n$.

Finally, for fixed $t \in[0, T]$, the sequence $\left(Y_{t}^{n}\right)$ is $\mathbb{P}$-almost surely non decreasing and the following convergence result holds:

$$
\mathbb{E} \sup _{t \in[0, T]}\left|Y_{t}^{n}-Y_{t}\right|^{2}+\mathbb{E} \int_{0}^{T}\left|Z_{t}^{n}-Z_{t}\right|^{2} d t+\mathbb{E}\left|K_{T}^{n}-K_{T}\right|^{2} \rightarrow 0 .
$$

Below we will need to show regular dependence of the solution to equations as (2.1) with respect to some parameters (for instance the initial data of a forward stochastic differential equation). Due to the generality of our assumptions on the nonlinearity $\psi$ this requires $L^{p}$ estimates both on the solution of equation (2.1) and on solutions of the penalized equations (2.3).

We assume the following:

Hypothesis 2.2 The function $f: \Omega \times[0, T] \times \mathbb{R} \times \Xi^{*} \rightarrow \mathbb{R}$ is measurable with respect to $\mathcal{P} \otimes \mathcal{B}(\mathbb{R}) \otimes \mathcal{B}\left(\Xi^{*}\right)$ and Lipschitz with respect to $y$ and $z$ uniformly in $t$ and $\omega$.

Moreover there exists $p \geq 2$ such that:

$$
\mathbb{E} \int_{0}^{T}|f(t, 0,0)|^{p}<\infty
$$

and the final data $\xi$ is in $L^{p}\left(\Omega, \mathcal{F}_{T}, \mathbb{P}\right)$.

Finally the obstacle $S$ is an adapted continuous real valued process satisfying

$$
\mathbb{E} \sup _{t \in[0, T]}\left|S_{t}\right|^{2 p-2}<\infty
$$


We notice that the integrability requests are not optimal (for instance we assume $p$-integrability jointly in $\Omega \times[0, T])$ for the generator $f$ and $2(p-1)$ integrability for the obstacle $S)$. Nevertheless such assumptions are verified in the Markovian framework (see Section 2.1) and will allow us to treat obstacle problems under general assumptions (see Section 3).

We claim that under Hypotheses 2.2 we can estimate the $L^{p}$ norms of the solution $(Y, Z, K)$ of equation (2.1). Namelly

Theorem 2.3 Let Hypothesis 2.2 hold and let $(Y, Z, K)$ be the unique mild solution to equation (2.1) then the following holds:

$$
\begin{aligned}
& \mathbb{E} \sup _{t \in[0, T]}\left|Y_{t}\right|^{p}+\mathbb{E}\left(\int_{0}^{T}\left|Z_{t}\right|^{2} d t\right)^{p / 2}+\mathbb{E}\left|K_{T}\right|^{p} \\
& \leq C \mathbb{E}|\xi|^{p}+C \mathbb{E} \int_{0}^{T}|f(t, 0,0)|^{p} d t+C\left(\mathbb{E} \sup _{t \in[0, T]}\left|S_{t}\right|^{2 p-2}\right)^{p /(2 p-2)},
\end{aligned}
$$

(we notice that $C$ in the above statement only depends on $T$ and on $f$ ).

The above result will be the consequence of the corresponding estimates for the penalized approximations.

Proposition 2.4 Let Hypothesis 2.2 hold and, for all $n \in \mathbb{N}$, let $\left(Y^{n}, Z^{n}\right)$ be the unique mild solution of equation (2.3) then the following holds:

$$
\begin{aligned}
& \mathbb{E} \sup _{t \in[0, T]}\left|Y_{t}^{n}\right|^{p}+\mathbb{E}\left(\int_{0}^{T}\left|Z_{t}^{n}\right|^{2} d t\right)^{p / 2}+\mathbb{E}\left|K_{T}^{n}\right|^{p} \\
& \leq C \mathbb{E}|\xi|^{p}+C \mathbb{E} \int_{0}^{T}|f(t, 0,0)|^{p} d t+C\left(\mathbb{E} \sup _{t \in[0, T]}\left|S_{t}\right|^{2 p-2}\right)^{p /(2 p-2)},
\end{aligned}
$$

where $C$ only depends on $p, T$ and on the Lipschitz constant of $f$ (recall that $\left.K_{t}^{n}=n \int_{0}^{t}\left(Y_{s}^{n}-S_{s}\right)^{-} d s\right)$.

Proof. First of all we notice that we can always reduce ourselves to the case in which

$$
\frac{y}{|y|} f(t, y, z) \leq|f(t, 0,0)|+\mu|y|+\lambda|z| \quad \text { with } \mu+\lambda^{2} \leq-1 .
$$

Indeed, setting $\tilde{Y}_{t}^{n}=e^{a t} Y_{t}^{n}, \tilde{Z}_{t}^{n}=e^{a t} Z_{t}^{n}, \tilde{S}_{t}=e^{a t} S_{t}$ we get that $\left(\tilde{Y}^{n}, \tilde{Z}^{n}\right)$ satisfies

$$
\left\{\begin{array}{c}
\left.-d \tilde{Y}_{t}^{n}=e^{a t} f\left(t, e^{-a t} \tilde{Y}_{t}^{n}, e^{-a t} \tilde{Z}_{t}^{n}\right) d t-a \tilde{Y}_{t}^{n} d t+n\left(\tilde{Y}_{t}^{n}-\tilde{S}_{t}\right)\right)^{-} d t \\
\quad-\tilde{Z}_{t}^{n} d W_{t}, \quad t \in[0, T], \\
\tilde{Y}_{T}^{n}=e^{a T} \xi
\end{array}\right.
$$

So the generator is given by

$$
\tilde{f}(t, y, z):=e^{a t} f\left(t, e^{-a t} y, e^{-a t} z\right)-a y
$$

and, by choosing a sufficiently large (depending only on the Lipscitz constant of $f$ ), we can assume $\mu+\lambda^{2} \leq-1$. From now on we assume that (2.8) holds true and for simplicity we omit the superscript $\sim$ where necessary. 
Moreover by $c$ we shall denote a constant that depends only on the Lipschitz constant of $f, T$ and $p$ and by $c(\delta)$ a constant that depends, beside the above parameters, on an auxiliary constant $\delta>0$. The values of $c$ and $c(\delta)$ can change from line to line. Finally to shorten notation we set:

$$
\Theta:=\mathbb{E}|\xi|^{p}+\mathbb{E} \int_{0}^{T}|f(t, 0,0)|^{p} d t+\left(\mathbb{E} \sup _{t \in[0, T]}\left|S_{t}\right|^{2 p-2}\right)^{p /(2 p-2)} .
$$

To start with, we apply Itô formula to $\left|Y_{t}^{n}\right|^{p}, s \leq t \leq T$ obtaining:

$$
\begin{aligned}
-d\left|Y_{t}^{n}\right|^{p}= & p\left|Y_{t}^{n}\right|^{p-1} \hat{Y}_{t}^{n} f\left(t, Y_{t}^{n}, Z_{t}^{n}\right) d t+p n\left|Y_{t}^{n}\right|^{p-1} \hat{Y}_{t}^{n}\left(Y_{t}^{n}-S_{t}\right)^{-} d t \\
& -p\left|Y_{t}^{n}\right|^{p-1} \hat{Y}_{t}^{n} Z_{t}^{n} d W_{t}-\frac{p(p-1)}{2}\left|Y_{t}^{n}\right|^{p-2}\left|Z_{t}^{n}\right|^{2} d t .
\end{aligned}
$$

where $\hat{Y}_{t}^{n}:=\frac{Y_{t}^{n}}{\left|Y_{t}^{n}\right|}$. Integrating between $s$ and $T, 0 \leq s \leq t \leq T$, we get:

$$
\begin{aligned}
& \left|Y_{s}^{n}\right|^{p}+\frac{p(p-1)}{2} \int_{s}^{T}\left|Y_{t}^{n}\right|^{p-2}\left|Z_{t}^{n}\right|^{2} d t \\
& =|\xi|^{p}+p \int_{s}^{T}\left|Y_{t}^{n}\right|^{p-1} \hat{Y}_{t}^{n} f\left(t, Y_{t}^{n}, Z_{t}^{n}\right) d t+n p \int_{s}^{T}\left|Y_{t}^{n}\right|^{p-1} \hat{Y}_{t}^{n}\left(Y_{t}^{n}-S_{t}\right)^{-} d t \\
& -p \int_{s}^{T}\left|Y_{t}^{n}\right|^{p-1} \hat{Y}_{t}^{n} Z_{t}^{n} d W_{t} \\
& \leq|\xi|^{p}+p \int_{s}^{T}\left|Y_{t}^{n}\right|^{p-1}|f(t, 0,0)| d t+p \mu \int_{s}^{T}\left|Y_{t}^{n}\right|^{p} d t+p \lambda \int_{s}^{T}\left|Y_{t}^{n}\right|^{p-1}\left|Z_{t}^{n}\right| d t \\
& +n p \int_{s}^{T}\left|S_{t}\right|^{p-1}\left(Y_{t}^{n}-S_{t}\right)^{-} d t-p \int_{s}^{T}\left|Y_{r}^{n}\right|^{p-1} \hat{Y}_{t}^{n} Z_{t}^{n} d W_{t} \\
& \leq|\xi|^{p}+c \int_{t}^{T}|f(t, 0,0)|^{p} d t+p \int_{s}^{T}\left|Y_{t}^{n}\right|^{p}+p \mu \int_{s}^{T}\left|Y_{t}^{n}\right|^{p} d t+\frac{p \lambda^{2}}{(p-1)} \int_{s}^{T}\left|Y_{t}^{n}\right|^{p} d t \\
& +\frac{p(p-1)}{4} \int_{s}^{T}\left|Y_{t}^{n}\right|^{p-2}\left|Z_{t}^{n}\right|^{2} d t+\sup _{t \in[s, T]}\left|S_{t}\right|^{p-1} n p \int_{s}^{T}\left(Y_{t}^{n}-S_{t}\right)^{-} d t \\
& -p \int_{s}^{T}\left|Y_{r}^{n}\right|^{p-1} \hat{Y}_{t}^{n} Z_{t}^{n} d W_{t},
\end{aligned}
$$

where we have applied Young inequality. So recalling that by (2.8) $\mu+\lambda^{2}+1 \leq 0$ (and consequently $p+p \mu+p(p-1)^{-1} \lambda^{2} \leq 0$, since $p \geq 2$ ) we get:

$$
\begin{aligned}
& \left|Y_{s}^{n}\right|^{p}+\frac{p(p-1)}{4} \int_{s}^{T}\left|Y_{t}^{n}\right|^{p-2}\left|Z_{t}^{n}\right|^{2} d t \\
& \leq|\xi|^{p}+c \int_{t}^{T}|f(t, 0,0)|^{p} d t \\
& +p \sup _{t \in[s, T]}\left|S_{t}\right|^{p-1} n \int_{s}^{T}\left(Y_{t}^{n}-S_{t}\right)^{-} d t-c \int_{s}^{T}\left|Y_{r}^{n}\right|^{p-1} \hat{Y}_{t}^{n} Z_{t}^{n} d W_{t} .
\end{aligned}
$$

By the penalized BSDE (2.3) in integral form we deduce that

$$
\int_{s}^{T} n\left(Y_{t}^{n}-S_{t}\right)^{-} d t=-\xi+Y_{t}^{n}-\int_{s}^{T} f\left(t, Y_{t}^{n}, Z_{t}^{n}\right) d t+\int_{s}^{T} Z_{t}^{n} d W_{t}
$$


therefore

$$
\begin{aligned}
& \left|Y_{s}^{n}\right|^{p}+\frac{p(p-1)}{4} \int_{s}^{T}\left|Y_{t}^{n}\right|^{p-2}\left|Z_{t}^{n}\right|^{2} d t \\
& \leq|\xi|^{p}+c \int_{s}^{T}|f(t, 0,0)|^{p} d t-p \int_{s}^{T}\left|Y_{r}^{n}\right|^{p-1} \hat{Y}_{t}^{n} Z_{t}^{n} d W_{t} \\
& +p\left[\sup _{t \in[s, T]}\left|S_{t}\right|^{p-1}\left(-\xi+Y_{s}^{n}-\int_{s}^{T} f\left(t, Y_{t}^{n}, Z_{t}^{n}\right) d t+\int_{s}^{T} Z_{t}^{n} d W_{t}\right)\right] \\
& \leq|\xi|^{p}+c \int_{s}^{T}|f(t, 0,0)|^{p} d t-p \int_{s}^{T}\left|Y_{r}^{n}\right|^{p-1} \hat{Y}_{t}^{n} Z_{t}^{n} d W_{t}+c \sup _{t \in[s, T]}\left|S_{t}\right|^{p}+\frac{1}{2}\left|Y_{s}^{n}\right|^{p} \\
& +p \sup _{t \in[s, T]}\left|S_{t}\right|^{p-1}\left[|\xi|+\left|\int_{s}^{T} f\left(t, Y_{t}^{n}, Z_{t}^{n}\right) d t\right|+\left|\int_{s}^{T} Z_{t}^{n} d W_{t}\right|\right]
\end{aligned}
$$

and moving the term $1 / 2\left|Y_{s}^{n}\right|^{p}$ to the left hand side:

$$
\begin{aligned}
& \frac{1}{2}\left|Y_{s}^{n}\right|^{p}+\frac{p(p-1)}{4} \int_{s}^{T}\left|Y_{t}^{n}\right|^{p-2}\left|Z_{t}^{n}\right|^{2} d t \\
& \leq|\xi|^{p}+c \int_{s}^{T}|f(t, 0,0)|^{p} d t-p \int_{s}^{T}\left|Y_{r}^{n}\right|^{p-1} \hat{Y}_{t}^{n} Z_{t}^{n} d W_{t}+c \sup _{t \in[s, T]}\left|S_{t}\right|^{p} \\
& +p \sup _{t \in[s, T]}\left|S_{t}\right|^{p-1}\left[|\xi|+\left|\int_{s}^{T} f\left(t, Y_{t}^{n}, Z_{t}^{n}\right) d t\right|+\left|\int_{s}^{T} Z_{t}^{n} d W_{t}\right|\right] .
\end{aligned}
$$

Since $\left(Y^{n}, Z^{n}\right) \in L_{\mathcal{P}}^{p}(\Omega, C([0, T])) \times L_{\mathcal{P}}^{p}\left(\Omega, L^{2}([0, T], \Xi)\right)$ (see [10]), the Itô integral $\int_{s}^{T}\left|Y_{r}^{n}\right|^{p-1} \hat{Y}_{t}^{n} Z_{t}^{n} d W_{t}$ has null expectation. Consequently, computing the mean value and recalling that, by Holder inequality:

$$
\mathbb{E} \sup _{t \in[s, t]}\left|S_{t}\right|^{p} \leq\left(\mathbb{E} \sup _{t \in[s, t]}\left|S_{t}\right|^{2 p-2}\right)^{p /(2 p-2)},
$$

we deduce that:

$$
\begin{aligned}
& \frac{1}{2} \mathbb{E}\left|Y_{s}^{n}\right|^{p}+\frac{p(p-1)}{4} \mathbb{E} \int_{s}^{T}\left|Y_{t}^{n}\right|^{p-2}\left|Z_{t}^{n}\right|^{2} d t \leq c \Theta \\
& +p \mathbb{E} \sup _{t \in[s, T]}\left|S_{t}\right|^{p-1}\left[|\xi|+\left|\int_{s}^{T} f\left(t, Y_{t}^{n}, Z_{t}^{n}\right) d t\right|+\left|\int_{s}^{T} Z_{t}^{n} d W_{t}\right|\right] \\
& \leq c \Theta+p\left(\mathbb{E} \sup _{t \in[s, T]}\left|S_{t}\right|^{2(p-1)}\right)^{\frac{1}{2}}\left(\mathbb{E}\left[|\xi|+\int_{s}^{T}\left(|f(t, 0,0)|+c\left|Y_{t}^{n}\right|+c\left|Z_{t}^{n}\right|\right) d t+\left|\int_{s}^{T} Z_{t}^{n} d W_{t}\right|\right]^{2}\right)^{\frac{1}{2}} .
\end{aligned}
$$

Plugging (2.4) in (2.11) we get, by the BDG inequality:

$$
\begin{aligned}
& \frac{1}{2} \mathbb{E}\left|Y_{s}^{n}\right|^{p}+\frac{p(p-1)}{4} \mathbb{E} \int_{s}^{T}\left|Y_{t}^{n}\right|^{p-2}\left|Z_{t}^{n}\right|^{2} d t \leq c \Theta \\
& +c\left(\mathbb{E} \sup _{t \in[s, T]}\left|S_{t}\right|^{2(p-1)}\right)^{\frac{1}{2}} \mathbb{E}\left[|\xi|^{2}+\left.\left.\left|\int_{0}^{T}\right| f(t, 0,0)\right|^{2} d t\left|+\int_{s}^{T}\right| Y_{t}^{n}\right|^{2}+\int_{s}^{T}\left|Z_{t}^{n}\right|^{2} d t\right]^{\frac{1}{2}} \\
& \leq c \Theta+c\left(\mathbb{E} \sup _{t \in[0, T]}\left|S_{t}\right|^{2(p-1)}\right)^{\frac{1}{2}} *\left(\mathbb{E}|\xi|^{2}+c \mathbb{E} \int_{0}^{T}|f(t, 0,0)|^{2} d t\right)^{\frac{1}{2}} .
\end{aligned}
$$


In particular

$$
\begin{aligned}
& \frac{p(p-1)}{4} \mathbb{E} \int_{s}^{T}\left|Y_{t}^{n}\right|^{p-2}\left|Z_{t}^{n}\right|^{2} d t \\
& \leq c \Theta+c\left(\mathbb{E} \sup _{t \in[0, T]}\left|S_{t}\right|^{2(p-1)}\right)^{\frac{1}{2}} *\left(\mathbb{E}|\xi|^{2}+C \mathbb{E} \int_{0}^{T}|f(t, 0,0)|^{2} d t\right)^{\frac{1}{2}}
\end{aligned}
$$

By (2.10) with $r$ instead of $s$, such that $0 \leq s \leq r \leq T$ we get (after multiplication by 2)

$$
\begin{aligned}
\left|Y_{r}^{n}\right|^{p} & \leq 2|\xi|^{p}+c \int_{0}^{T}|f(t, 0,0)|^{p} d t \\
& -2 p \int_{r}^{T}\left|Y_{r}^{n}\right|^{p-1} \hat{Y}_{t}^{n} Z_{t}^{n} d W_{t}+c \sup _{r \in[s, T]}\left|S_{t}\right|^{p}+ \\
& +2 p \sup _{r \in[s, T]}\left|S_{r}\right|^{p-1}\left[|\xi|+\left|\int_{r}^{T} f\left(t, Y_{t}^{n}, Z_{t}^{n}\right) d t\right|+\left|\int_{r}^{T} Z_{t}^{n} d W_{t}\right|\right] .
\end{aligned}
$$

By computing the supremum over the time $r$ we arrive at:

$$
\begin{aligned}
& \mathbb{E} \sup _{r \in[s, T]}\left|Y_{r}^{n}\right|^{p} \\
& \leq c \Theta+\left.\mathbb{E} \sup _{r \in[s, T]}\left|\int_{r}^{T}\right| Y_{t}^{n}\right|^{p-1} \hat{Y}_{t}^{n} Z_{t}^{n} d W_{t} \mid \\
& +2\left(\mathbb{E} \sup _{t \in[s, T]}\left|S_{t}\right|^{2(p-1)}\right)^{\frac{1}{2}}\left(\mathbb{E}\left[|\xi|^{2}+\int_{s}^{T}\left|f\left(t, Y_{t}^{n}, Z_{t}^{n}\right)\right|^{2} d t+\sup _{t \in[s, T]}\left|\int_{t}^{T} Z_{t}^{n} d W_{t}\right|^{2}\right]\right)^{\frac{1}{2}}
\end{aligned}
$$

Proceeding as in th proof of (2.12) we get:

$$
\begin{aligned}
& \mathbb{E} \sup _{r \in[s, T]}\left|Y_{r}^{n}\right|^{p} \leq c \Theta+c \mathbb{E} \sup _{r \in[s, T] \mid} \int_{r}^{T}\left|Y_{t}^{n}\right|^{p-1} \hat{Y}_{t}^{n} Z_{t}^{n} d W_{t} \mid \\
& +c\left(\mathbb{E} \sup _{t \in[s, T]}\left|S_{t}\right|^{2(p-1)}\right)^{\frac{1}{2}}\left(\mathbb{E}\left[|\xi|^{2}+\int_{s}^{T}|f(t, 0,0)|^{2} d t\right]\right)^{\frac{1}{2}}
\end{aligned}
$$

and, again by BDG inequality:

$$
\begin{aligned}
& \mathbb{E} \sup _{r \in[s, T]}\left|Y_{r}^{n}\right|^{p} \leq c \mathbb{E}|\xi|^{p}+c \int_{s}^{T}|f(t, 0,0)|^{p} d t+c \mathbb{E}\left(\int_{r}^{T}\left|Y_{t}^{n}\right|^{2(p-1)}\left|Z_{t}^{n}\right|^{2} d t\right)^{\frac{1}{2}} \\
& +c\left(\mathbb{E} \sup _{t \in[s, T]}\left|S_{t}\right|^{2(p-1)}\right)^{\frac{1}{2}}\left(\mathbb{E}\left[|\xi|^{2}+\int_{s}^{T}|f(t, 0,0)|^{2} d t\right]\right)^{\frac{1}{2}} .
\end{aligned}
$$

Therefore

$$
\begin{aligned}
& \mathbb{E} \sup _{r \in[s, T]}\left|Y_{r}^{n}\right|^{p} \leq c \Theta+c \mathbb{E}\left(\sup _{t \in[s, T]}\left|Y_{t}^{n}\right|^{p} \int_{s}^{T}\left|Y_{t}^{n}\right|^{p-2}\left|Z_{t}^{n}\right|^{2} d t\right)^{\frac{1}{2}} \\
& +c\left(\mathbb{E} \sup _{t \in[s, T]}\left|S_{t}\right|^{2(p-1)}\right)^{\frac{1}{2}}\left(\mathbb{E}\left[|\xi|^{2}+\int_{s}^{T}|f(t, 0,0)|^{2} d t\right]\right)^{\frac{1}{2}},
\end{aligned}
$$


and finally

$$
\begin{aligned}
& \mathbb{E} \sup _{r \in[s, T]}\left|Y_{r}^{n}\right|^{p} \leq c \Theta+\frac{1}{2} \mathbb{E} \sup _{r \in[s, T]}\left|Y_{r}^{n}\right|^{p}+c \mathbb{E} \int_{s}^{T}\left|Y_{t}^{n}\right|^{p-2}\left|Z_{t}^{n}\right|^{2} d t \\
& +c\left(\mathbb{E} \sup _{t \in[s, T]}\left|S_{t}\right|^{2(p-1)}\right)^{\frac{1}{2}}\left(\mathbb{E}\left[|\xi|^{2}+\int_{s}^{T}|f(t, 0,0)|^{2} d t \mid\right]\right)^{\frac{1}{2}} .
\end{aligned}
$$

Applying estimate (2.13) we can conclude:

$$
\mathbb{E} \sup _{r \in[s, T]}\left|Y_{r}^{n}\right|^{p} \leq c \Theta+\left(\mathbb{E} \sup _{t \in[s, T]}\left|S_{t}\right|^{2(p-1)}\right)^{\frac{1}{2}}\left(\mathbb{E}\left[|\xi|^{2}+\int_{s}^{T}|f(t, 0,0)|^{2} d t\right]\right)^{\frac{1}{2}}
$$

Next we estimate $\mathbb{E}\left(\int_{s}^{T}\left|Z_{t}^{n}\right|^{2} d t\right)^{\frac{p}{2}}$. We apply Itô formula to $\left|Y_{t}^{n}\right|^{2}, s \leq t \leq T$ and get:

$$
d\left|Y_{t}^{n}\right|^{2}=-2 Y_{t}^{n} f\left(t, Y_{t}^{n}, Z_{t}^{n}\right) d t-n Y_{t}^{n}\left(Y_{t}^{n}-S_{t}\right)^{-} d t+2 Y_{t}^{n} Z_{t}^{n} d W_{t}+\left|Z_{t}^{n}\right|^{2} d t .
$$

We integrate on $[s, T]$ and we raise to the power $\frac{p}{2}$ :

$$
\begin{aligned}
& \left|Y_{s}^{n}\right|^{p}+\left(\int_{s}^{T}\left|Z_{t}^{n}\right|^{2} d t\right)^{\frac{p}{2}} \\
& \leq|\xi|^{p}+c\left|\int_{s}^{T} Y_{t}^{n} f\left(t, Y_{t}^{n}, Z_{t}^{n}\right) d t\right|^{\frac{p}{2}}+c\left|n \int_{s}^{T} Y_{t}^{n}\left(Y_{t}^{n}-S_{t}\right)^{-} d t\right|^{\frac{p}{2}} \\
& +c\left|\int_{s}^{T} Y_{r}^{n} Z_{t}^{n} d W_{t}\right|^{\frac{p}{2}} \\
& \leq|\xi|^{p}+c\left|\int_{s}^{T} Y_{t}^{n} f\left(t, Y_{t}^{n}, Z_{t}^{n}\right) d t\right|^{\frac{p}{2}}+c\left(\sup _{t \in[s, T]} n\left|S_{t}\right|\left|\int_{s}^{T} Y_{t}^{n}\left(Y_{t}^{n}-h\left(X_{t}\right)\right)^{-} d t\right|\right)^{\frac{p}{2}} \\
& +c\left|\int_{s}^{T} Y_{r}^{n} Z_{t}^{n} d W_{t}\right|^{\frac{p}{2}} \cdot
\end{aligned}
$$

Using the expression (2.9) for $n \int_{s}^{T}\left(Y_{t}^{n}-S_{t}\right)^{-} d t$ we get:

$$
\begin{aligned}
& \left(\int_{s}^{T}\left|Z_{t}^{n}\right|^{2} d t\right)^{\frac{p}{2}} \\
& \leq|\xi|^{p}+c\left|\int_{s}^{T} Y_{t}^{n} f\left(t, Y_{t}^{n}, Z_{t}^{n}\right) d t\right|^{\frac{p}{2}}+c\left|\int_{s}^{T} Y_{t}^{n} Z_{t}^{n} d W_{t}\right|^{\frac{p}{2}} \\
& +c \sup _{t \in[s, T]}\left|S_{t}\right|^{\frac{p}{2}}\left|-\xi+Y_{t}^{n}-\int_{s}^{T} f\left(t, Y_{t}^{n}, Z_{t}^{n}\right) d t+\int_{s}^{T} Z_{t}^{n} d W_{t}\right|^{\frac{p}{2}} \\
& \leq|\xi|^{p}+c\left(\int_{s}^{T}\left(\left|Y_{t}^{n}\right||f(t, 0,0)|+\mu\left|Y_{t}^{n}\right|^{2}+\lambda\left|Y_{t}^{n}\right|\left|Z_{t}^{n}\right|\right) d t\right)^{\frac{p}{2}}+c\left|\int_{s}^{T} Y_{t}^{n} Z_{t}^{n} d W_{t}\right|^{\frac{p}{2}} \\
& +c \sup _{t \in[s, T]}\left|S_{t}\right|^{\frac{p}{2}}\left(|\xi|+\left|Y_{t}^{n}\right|+\int_{s}^{T}\left(|f(t, 0,0)|+\mu\left|Y_{t}^{n}\right|+\lambda\left|Z_{t}^{n}\right|\right) d t+\left|\int_{s}^{T} Z_{t}^{n} d W_{t}\right|\right)^{\frac{p}{2}} .
\end{aligned}
$$


Computing expectation, applying BDG and Young inequalities and estimate (2.14), we have:

$$
\begin{aligned}
& \mathbb{E}\left(\int_{s}^{T}\left|Z_{t}^{n}\right|^{2} d t\right)^{\frac{p}{2}} \\
& \leq \mathbb{E}|\xi|^{p}+c \mathbb{E} \sup _{r \in[s, T]}\left|Y_{r}^{n}\right|^{p}+c \mathbb{E}\left(\int_{s}^{T}|f(t, 0,0)|^{2} d t\right)^{\frac{p}{2}} \\
& +\frac{1}{4}\left(\mathbb{E} \int_{s}^{T}\left|Z_{t}^{n}\right|^{2} d t\right)^{\frac{p}{2}}+c \mathbb{E}\left(\int_{s}^{T}\left|Y_{t}^{n} Z_{t}^{n}\right|^{2} d t\right)^{\frac{p}{4}}+c \mathbb{E} \sup _{t \in[s, T]}\left|S_{t}\right|^{p} \\
& \leq c \Theta+\frac{1}{2} \mathbb{E}\left(\int_{s}^{T}\left|Z_{t}^{n}\right|^{2} d t\right)^{\frac{p}{2}}+c \mathbb{E} \sup _{r \in[s, T]}\left|Y_{r}^{n}\right|^{p} .
\end{aligned}
$$

Finally by estimate (2.14), we obtain:

$$
\mathbb{E}\left(\int_{s}^{T}\left|Z_{t}^{n}\right|^{2} d t\right)^{\frac{p}{2}} \leq c \Theta+c\left(\mathbb{E} \sup _{t \in[s, T]}\left|S_{t}\right|^{2(p-1)}\right)^{\frac{1}{2}}\left(\mathbb{E}\left[|\xi|^{2}+\left.\left|\int_{s}^{T}\right| f(t, 0,0)\right|^{2} d t \mid\right]\right)^{\frac{1}{2}}
$$

and this concludes the proof of the estimate of $\mathbb{E}\left(\int_{s}^{T}\left|Z_{t}^{n}\right|^{2} d t\right)^{\frac{p}{2}}$.

The estimate of $\mathbb{E}\left|K_{T}^{n}\right|^{p}$ is now an easy consequence of the previous ones and of relation (2.9).

We are now ready to prove Theorem 2.3.

\section{Proof of Theorem 2.3.}

We already know (see Theorem 2.1) that $Y_{t}^{n} \uparrow Y_{t}$ and $\mathbb{E}\left(\sup _{t \in[0, T]}\left(Y_{t}-Y_{t}^{n}\right)\right)^{2} \rightarrow 0$.

Choosing a suitable subsequence we can assume that $\sup _{t \in[0, T]}\left(Y_{t}-Y_{t}^{n}\right) \rightarrow 0, \mathbb{P}$-almost surely. Therefore, Fatou Lemma and (2.7) yield:

$$
\mathbb{E} \sup _{t \in[0, T]}\left|Y_{t}\right|^{p} \leq C \mathbb{E}|\xi|^{p}+C \mathbb{E} \int_{0}^{T}|f(t, 0,0)|^{p} d t+C\left(\mathbb{E} \sup _{t \in[0, T]}\left|S_{t}\right|^{2 p-2}\right)^{p /(2 p-2)} .
$$

As far as the convergence of $Z^{n}$ is concerned, we already know that $Z^{n} \rightarrow Z$ in $L_{\mathcal{P}}^{2}(\Omega \times[0, T])$, see (2.5). By Proposition 2.4 we know that $Z^{n}$ is bounded in $L_{\mathcal{P}}^{p}(\Omega \times[0, T])$, so, extracting, if needed, a subsequence, we can assume that $\left(Z^{n}\right)$ converges weakly in $L_{\mathcal{P}}^{p}(\Omega \times[0, T])$ and consequently also weakly in $L_{\mathcal{P}}^{2}(\Omega \times[0, T])$. Therefore the weak limit of $\left(Z^{n}\right)$ in $L_{\mathcal{P}}^{p}(\Omega \times[0, T])$ must coincide with the strong limit $Z$ in $L_{\mathcal{P}}^{2}(\Omega \times[0, T])$ topology. Consequently again by $(2.7)$ we have that $Z$ satisfies

$$
\mathbb{E}\left(\int_{0}^{T}\left|Z_{t}\right|^{2} d t\right)^{p / 2} \leq C \mathbb{E}|\xi|^{p}+C \mathbb{E} \int_{0}^{T}|f(t, 0,0)|^{p} d t+C\left(\mathbb{E} \sup _{t \in[0, T]}\left|S_{t}\right|^{2 p-2}\right)^{p /(2 p-2)} .
$$

To prove the convergence of processes $K^{n}$ we argue in a similar way. By (2.5) we know that $\mathbb{E}\left|K_{T}^{n}-K_{T}\right|^{2} \rightarrow 0$. The claim follows as before by Fatou lemma exploiting estimate (2.7). 


\subsection{Reflected BSDEs in a Markovian framework}

Now we consider a RBSDE depending on a forward equation with values in another real and separable Hilbert space $H$. Namely, we consider the forward backward system

$$
\left\{\begin{array}{l}
d X_{t}^{s, x}=A X_{t}^{s, x} d t+F\left(t, X_{t}^{s, x}\right) d t+G\left(t, X_{t}^{s, x}\right) d W_{t} \quad t \in[s, T] \\
X_{s}^{s, x}=x, \\
-d Y_{t}^{s, x}=\psi\left(t, X_{t}^{s, x}, Y_{t}^{s, x}, Z_{t}^{s, x}\right) d t+d K_{t}^{s, x}-Z_{t}^{s, x} d W_{t}, \quad t \in[s, T], \\
Y_{T}^{s, x}=\phi\left(X_{T}^{s, x}\right), \\
Y_{t}^{s, x} \geq h\left(X_{t}^{s, x}\right) \\
\int_{s}^{T}\left(Y_{t}^{s, x}-h\left(X_{t}^{s, x}\right)\right) d K_{t}^{s, x}=0 .
\end{array}\right.
$$

We denote the solution of the RBSDE in the above equation by $\left(Y^{s, x}, Z^{s, x}, K^{s, x}\right)$, to stress the dependence on the initial conditions, or by $(Y, Z, K)$ if no confusion is possible.

Below and in the rest of the paper we will use the following notation:

- if $K_{1}$ and $K_{2}$ are Hilbert spaces by $L_{2}\left(K_{1}, K_{2}\right)$ we denote the Hilbert space of HilbertSchmidt operators $K_{1} \rightarrow K_{2}$ endowed with the Hilbert-Schmidt norm;

- by $\mathcal{G}\left(K_{1}, K_{2}\right)$ we denote the space of all continuously Gateaux differentiable mappings from $K_{1}$ to $K_{2}$. See [10] for details.

We will work under the following assumptions on the coefficients of the forward equation:

Hypothesis 2.5 $\quad$ 1. $A: H \supset \mathcal{D}(A) \rightarrow H$ is the generator of a strongly continuous semigroup of linear operators $\left(e^{t A}\right)_{t \geq 0}$.

2. The mapping $F:[0, T] \times H \rightarrow H$ is measurable and satisfies, for some constant $C>0$ and $0 \leq \gamma<1$,

$$
\begin{aligned}
& \left|e^{s A} F(\tau, x)\right| \leq C s^{-\gamma}(1+|x|), \quad t \in[0, T], \\
& \left|e^{s A} F(\tau, x)-e^{s A} F(\tau, y)\right| \leq C s^{-\gamma}|x-y|, \quad s>0, t \in[0, T], \quad x, y \in H .
\end{aligned}
$$

3. $G$ is a mapping $[0, T] \times H \rightarrow L(\Xi, H)$ such that for every $v \in \Xi$, the map $G v:[0, T] \times H \rightarrow$ $H$ is measurable and for every $s>0, \tau \in[0, T]$ and $x \in H$ we have $e^{s A} G(\tau, x) \in L_{2}(\Xi, H)$. Moreover there exists $0<\theta<\frac{1}{2}$ such that

$$
\begin{aligned}
& \left|e^{s A} G(\tau, x)\right|_{L_{2}(\Xi, H)} \leq L s^{-\theta}(1+|x|), \\
& \left|e^{s A} G(\tau, x)-e^{s A} G(\tau, y)\right|_{L_{2}(\Xi, H)} \leq L s^{-\theta}|x-y|, \quad s>0, \tau \in[0, T], \quad x, y \in H .
\end{aligned}
$$

4. For every $s>0, t \in[0, T], F(t, \cdot) \in \mathcal{G}(H, H)$ and $e^{s A} G(t, \cdot) \in \mathcal{G}\left(H, L_{2}(\Xi, H)\right)$.

The next existence and uniqueness Proposition is proved in [10].

Proposition 2.6 Under Hypothesis 2.5, the forward equation

$$
\left\{\begin{array}{l}
d X_{t}^{s, x}=A X_{t}^{s, x} d t+F\left(t, X_{t}^{s, x}\right) d t+G\left(t, X_{t}^{s, x}\right) d W_{t} \quad t \in[s, T] \\
X_{s}^{s, x}=x
\end{array}\right.
$$

admits a unique continuous mild solution. Moreover $\mathbb{E} \sup _{t \in[s, T]}\left|X_{t}^{s, x}\right|^{p} \leq C_{p}(1+|x|)^{p}$, for every $p \in(0, \infty)$, and some constant $C_{p}>0$. 
We will work under the following assumptions on $\psi$ :

Hypothesis 2.7 The function $\psi:[0, T] \times H \times \mathbb{R} \times \Xi^{*} \rightarrow \mathbb{R}$ is Borel measurable and satisfies the following:

1. there exists a constant $L>0$ such that

$$
\left|\psi\left(t, x, y_{1}, z_{1}\right)-\psi\left(t, x, y_{2}, z_{2}\right)\right| \leq L\left(\left|y_{1}-y_{2}\right|+\left|z_{1}-z_{2}\right|_{\Xi^{*}}\right),
$$

for every $t \in[0, T], x \in H, y_{1}, y_{2} \in \mathbb{R}, z_{1}, z_{2} \in \Xi^{*}$;

2. for every $t \in[0, T], \psi(t, \cdot, \cdot, \cdot)$ is continuous $H \times \mathbb{R} \times \Xi^{*} \rightarrow \mathbb{R}$;

3. there exists $L^{\prime}>0$ and $m \geq 0$ such that:

$$
\left|\psi\left(t, x_{1}, y, z\right)-\psi\left(t, x_{2}, y, z\right)\right| \leq L^{\prime}\left|x_{1}-x_{2}\right|\left(1+\left|x_{1}\right|^{m}+\left|x_{2}\right|^{m}+|y|^{m}\right)\left(1+|z|_{\Xi}\right),
$$

for every $t \in[0, T], x_{1}, x_{2} \in H, y \in \mathbb{R}, z \in \Xi$;

4. there exists $L>0$ such that:

$$
\begin{aligned}
& \left|\phi\left(x_{1}\right)-\phi\left(x_{2}\right)\right| \leq L\left|x_{1}-x_{2}\right|\left(1+\left|x_{1}\right|^{m}+\left|x_{2}\right|^{m}\right), \\
& \left|h\left(x_{1}\right)-h\left(x_{2}\right)\right| \leq L\left|x_{1}-x_{2}\right|\left(1+\left|x_{1}\right|^{m}+\left|x_{2}\right|^{m}\right),
\end{aligned}
$$

for all $x_{1}, x_{2} \in H$.

We notice that Hypothesis 2.7 implies that:

$|\psi(t, x, y, z)| \leq L\left(1+|x|^{m+1}+|y|+|z|_{\Xi^{*}}\right), \quad|\phi(x)| \leq L\left(1+|x|^{m+1}\right), \quad|h(x)| \leq L\left(1+|x|^{m+1}\right)$,

for all $t \in[0, T], x \in H, y \in \mathbb{R} z \in \Xi$.

Proposition 2.8 Let Hypotheses 2.5 and 2.7 hold true and fix $s \in[0, T], x \in H$. Then the $R B S D E$ in (2.15) admits a unique adapted solution $\left(Y^{s, x}, Z^{s, x}, K^{s, x}\right)$. Moreover $Y^{s, x}$ admits a continuous version, $\left(K^{s, x}\right)$ is continuous and non-decreasing $\left(K_{0}^{s, x}=0\right)$ and, for all $p \geq 2$ there exists $C_{p}>0$ such that

$$
\mathbb{E} \sup _{t \in[0, T]}\left|Y_{t}^{s, x}\right|^{p}+\mathbb{E}\left(\int_{0}^{T}\left|Z_{t}^{s, x}\right|^{2} d t\right)^{p / 2}+\mathbb{E}\left|K_{T}^{s, x}\right|^{p} \leq C\left(1+|x|^{p(m+1)}\right) .
$$

Moreover if we consider the penalized version of the RBSDE in (2.15):

$\left\{\begin{array}{l}-d Y_{t}^{n, s, x}=\psi\left(t, X_{t}^{s, x}, Y_{t}^{n, s, x}, Z_{t}^{n, s, x}\right) d t+n\left(Y_{t}^{n, s, x}-h\left(X_{t}^{s, x}\right)\right)^{-} d t-Z_{t}^{n, s, x} d W_{t}, \quad t \in[0, T], \\ Y_{T}^{n, s, x}=\phi\left(X_{T}^{s, x}\right) .\end{array}\right.$

then (2.20) holds for equation (2.21) as well (with constant $C$ independent on $n$ ).

Proof. It suffices to notice that if we set:

$$
f(t, y, z):=\psi\left(t, X_{t}^{s, x}, y, z\right), S_{t}:=h\left(X_{t}^{s, x}\right), \xi:=\phi\left(X_{T}^{s, x}\right)
$$


where $t \in[0, T], y \in \mathbb{R}, z \in \Xi^{*}$ and $X^{s, x}$ is the solution of equation(2.18), then, by (2.19), $f, h, S$ satisfy Hypothesis 2.2, and in particular:

$$
\begin{aligned}
& \mathbb{E} \int_{s}^{T}|f(t, 0,0)|^{p} d t=\mathbb{E} \int_{s}^{T}\left|\psi\left(t, X_{t}^{s, x}, 0,0\right)\right|^{p} d t \leq c\left(1+|x|^{p(m+1)}\right) \\
& \mathbb{E} \sup _{t \in[s, T]}\left|S_{t}\right|^{2(p-1)}=\mathbb{E} \sup _{t \in[s, T]}\left|h\left(X_{t}^{s, x}\right)\right|^{2(p-1)} \leq c\left(1+|x|^{2(p-1)(m+1)}\right) \\
& \mathbb{E}|\xi|^{p}=\left.\mathbb{E}|| \phi\left(X_{t}^{s, x}\right)\right|^{p} \leq\left(1+|x|^{p(m+1)}\right) .
\end{aligned}
$$

The claim follows by Proposition 2.4 and Theorem 2.3 .

Remark 2.9 We note that the processes $\left(X_{t}^{s, x}, Y_{t}^{s, x}, Z_{t}^{s, x}\right)_{t \geq s}$ are independent of $\mathcal{F}_{s}$.

For further use we also note some useful identities. The first one is the well-known flow property (see for instance [3]): for fixed $0 \leq r \leq s \leq T$ we have, $\mathbb{P}$-a.s.,

$$
X_{t}^{r, x}=X_{t}^{s, X_{s}^{r, x}}, \quad t \in[s, T] .
$$

Since the driving noises in the backward equation on the interval $[s, T]$ are the increments of the Wiener process and the values of the process $X$, by using the last identity it is easy to verify that the triples $\left(Y_{t}^{s, X_{s}^{r, x}}, Z_{t}^{s, X_{s}^{r, x}}, K_{t}^{s, X_{s}^{r, x}}\right)_{t \in[s, T]}$ and $\left(Y_{t}^{r, x}, Z_{t}^{r, x}, K_{t}^{r, x}\right)_{t \in[s, T]}$ are both solutions to the same backward equation on $[s, T]$. According to the uniqueness statement in Theorem 2.1, the first two components coincide as elements of the space $L_{\mathcal{P}}^{2}(\Omega, C([s, T])) \times L_{\mathcal{P}}^{2}\left(\Omega \times[s, T], \Xi^{*}\right)$ and we conclude that for fixed $0 \leq r \leq s \leq T$ we have, $\mathbb{P}$-a.s.,

$$
\begin{gathered}
Y_{t}^{s, X_{s}^{r, x}}=Y_{t}^{r, x}, \quad t \in[s, T], \\
Z_{t}^{s, X_{s}^{r, x}}=Z_{t}^{r, x}, \quad \text { for a.e. } t \in[s, T] .
\end{gathered}
$$

The next theorem is devoted to the local Lipschitz continuity of $Y^{s, x}$ with respect to $x$.

Theorem 2.10 Let Hypotheses 2.5 and 2.7 hold true and let $\left(Y^{s, x}, Z^{s, x}, K^{s, x}\right)$ be the unique solution of the the RBSDE in (2.15). Then there exists a constant $C>0$ such that, $\forall x_{1}, x_{2} \in H$,

$$
\left|Y_{s}^{s, x_{1}}-Y_{s}^{s, x_{2}}\right| \leq C\left(1+\left|x_{1}\right|^{m(m+1)}+\left|x_{2}\right|^{m(m+1)}\right)\left|x_{1}-x_{2}\right| .
$$

Proof. We initially assume that the generator $\psi$, the final datum $\psi$ and the obstacle $h$ are differentiable, namely for every $t \in[0, T]$ we assume that $\psi(t, \cdot, \cdot, \cdot) \in \mathcal{G}\left(H \times \mathbb{R} \times \Xi^{*}, \mathbb{R}\right), h(t, \cdot) \in$ $\mathcal{G}(H, \mathbb{R})$ and $\phi \in \mathcal{G}(H, \mathbb{R})$. The idea is to prove that, in the case of smooth (differentiable) coefficients, the solution of the penalized equation (2.21) is differentiable with respect to $x$, and the derivative is bounded uniformly with respect to $n$.

In order to work in a "smooth" framework, in the penalized BSDE (2.21) we have to replace the non-smooth penalizing term $n(y-h)^{-}$by $n \gamma(y-h)$ where $\gamma: \mathbb{R} \rightarrow \mathbb{R}^{1}$ is a function in $C_{b}^{1}(\mathbb{R})$ verifying:

$$
\begin{aligned}
& \gamma(y)=0 \text { for } y \geq 0, \quad \gamma(y)>0 \text { for } y<0 \\
& \gamma(y)=-y \text { for } y \leq-1, \quad \dot{\gamma}(y)<0 \text { for } y<0 .
\end{aligned}
$$


Notice that to construct $\gamma$ it is enough to set $\gamma(y)=\int_{0}^{-y} \ell(r) d r$ with

$$
\ell(r)=0 \text { for } r \leq 0, \quad \ell(r)>0 \text { for } r>0, \quad \ell(r)=1 \text { for } r \geq 1, \quad \int_{0}^{1} \ell(r) d r=1 .
$$

So we consider the following "smooth" penalized BSDE

$$
\left\{\begin{array}{l}
-d Y_{t}^{n, s, x}=\psi\left(t, X_{t}^{s, x}, Y_{t}^{n, s, x}, Z_{t}^{n, s, x}\right) d t+n \gamma\left(Y_{t}^{n, s, x}-h\left(X_{t}^{s, x}\right)\right) d t-Z_{t}^{n, s, x} d W_{t}, \quad t \in[0, T], \\
Y_{T}^{n, s, x}=\phi\left(X_{T}^{s, x}\right),
\end{array}\right.
$$

and we notice that estimates obtained in Proposition 2.8 are still true for the pair of processes $\left(Y^{n, s, x}, Z^{n, s, x}\right)$ solution of equation (2.25). Indeed, it is still true that $|y|^{p-1} \hat{y} \gamma(y-s) \leq$ $|s|^{p-1} \gamma(y-s)$ for all $y, s, \in \mathbb{R}$.

By [10] we know that we can differentiate $\left(Y^{n, s, x}, Z^{n, s, x}\right)$ with respect to $x$, and that $\left(\nabla_{x} Y^{n, s, x}\right.$, $\left.\nabla_{x} Z^{n, s, x}\right)$ is the solution of the following linear BSDE:

$$
\left\{\begin{aligned}
-d \nabla_{x} d Y_{t}^{n, s, x} & =\nabla_{x} \psi\left(t, X_{t}^{s, x}, Y_{t}^{n, s, x}, Z_{t}^{n, s, x}\right) \nabla_{x} X_{t}^{s, x} d t+\nabla_{y} \psi\left(t, X_{t}^{s, x}, Y_{t}^{n, s, x}, Z_{t}^{n, s, x}\right) \nabla_{x} Y_{t}^{n, s, x} d t \\
& +n \dot{\gamma}\left(Y_{t}^{n, s, x}-h\left(X_{t}^{s, x}\right)\right)\left(\nabla_{x} Y_{t}^{n, s, x}-\nabla h\left(X_{t}^{s, x}\right) \nabla_{x} X_{t}^{s, x}\right) d t \\
& +\nabla_{z} \psi\left(t, X_{t}^{s, x}, Y_{t}^{n, s, x}, Z_{t}^{n, s, x}\right) \nabla_{x} Z_{t}^{n, s, x} d t-\nabla_{x} Z_{t}^{n, s, x} d W_{t}, \quad t \in[s, T], \\
\nabla_{x} Y_{T}^{n, s, x}= & \nabla \phi\left(X_{T}^{s, x}\right) \nabla_{x} X_{T}^{s, x} .
\end{aligned}\right.
$$

where (see again [10]) $\nabla_{x} X^{s, x}$ is the mild solution to the following forward equation (to be intended in mild form):

$\left\{\begin{array}{l}d \nabla_{x} X_{t}^{s, x}=A \nabla_{x} X_{t}^{s, x} d t+\nabla_{x} F\left(t, X_{t}^{s, x}\right) \nabla_{x} X_{t}^{s, x} d t+\nabla_{x} G\left(t, X_{t}^{s, x}\right) \nabla_{x} X_{t}^{s, x} d W_{t}, \quad t \in[s, T] \\ \nabla_{x} X_{s}^{s, x}=I,\end{array}\right.$

$I: H \rightarrow H$ being the identity operator in $H$.

We set $\tilde{\mathbb{P}}:=\mathcal{E}_{T} \mathbb{P}$, with

$$
\mathcal{E}_{T}=\exp \left(\int_{s}^{T} \nabla_{z} \psi\left(t, X_{t}^{s, x}, Y_{t}^{n, s, x}, Z_{t}^{n, s, x}\right) d W_{t}-\frac{1}{2} \int_{s}^{T}\left|\nabla_{z} \psi\left(t, X_{t}^{s, x}, Y_{t}^{n, s, x}, Z_{t}^{n, s, x}\right)\right|^{2} d t\right) .
$$

By the Girsanov theorem $\tilde{\mathbb{P}}$ is a probability measure equivalent to the original one $\mathbb{P}$ (recall that by Hypothesis $2.7, \nabla_{z} \psi$ is bounded) and

$$
\tilde{W}_{\tau}=-\int_{s}^{\tau} \nabla_{z} \psi\left(t, X_{t}^{s, x}, Y_{t}^{n, s, x}, Z_{t}^{n, s, x}\right) d t+W_{\tau}, \quad s \leq \tau \leq T
$$

is a $\tilde{\mathbb{P}}_{\text {-cylindrical Wiener process. }}$

In $(\Omega, \mathcal{F}, \tilde{\mathbb{P}})$ the pair $\left(\nabla_{x} Y^{n, s, x}, \nabla_{x} Z^{n, s, x}\right)$ solves the following BSDE for $t \in[s, T]$ :

$$
\left\{\begin{aligned}
-d \nabla_{x} d Y_{t}^{n, s, x} & =\nabla_{x} \psi\left(t, X_{t}^{s, x}, Y_{t}^{n, s, x}, Z_{t}^{n, s, x}\right) \nabla_{x} X_{t}^{s, x} d t \\
& +\nabla_{y} \psi\left(t, X_{t}^{s, x}, Y_{t}^{n, s, x}, Z_{t}^{n, s, x}\right) \nabla_{x} Y_{t}^{n, s, x} d t \\
& +n \dot{\gamma}\left(Y_{t}^{n, s, x}-h\left(X_{t}^{s, x}\right)\right)\left(\nabla_{x} Y_{t}^{n, s, x}-\nabla h\left(X_{t}^{s, x}\right) \nabla_{x} X_{t}^{s, x}\right) d t-\nabla_{x} Z_{t}^{n, s, x} d \tilde{W}_{t}, \\
\nabla_{x} Y_{T}^{n, s, x}= & \nabla \phi\left(X_{T}^{s, x}\right) \nabla_{x} X_{T}^{s, x} .
\end{aligned}\right.
$$

Multiplying $\nabla_{x} Y_{t}^{n, s, x}$ by $\exp \left\{\int_{s}^{t}\left(\nabla_{y} \psi\left(t, X_{\sigma}^{s, x}, Y_{\sigma}^{n, s, x}, Z_{\sigma}^{n, s, x}\right)+n \dot{\gamma}\left(Y_{\sigma}^{n, s, x}-h\left(X_{\sigma}^{s, x}\right)\right)\right) d \sigma\right\}$ and 
choosing $t=s$ we get:

$$
\begin{aligned}
& \nabla_{x} Y_{s}^{n, s, x} \\
& =\mathbb{E}\left[\mathcal{E}_{T} \int_{s}^{T} \exp \left\{\int_{s}^{\tau} \nabla_{y} \psi\left(t, X_{\sigma}^{s, x}, Y_{\sigma}^{n, s, x}, Z_{\sigma}^{n, s, x}\right)+n \dot{\gamma}\left(Y_{\sigma}^{n, s, x}-h\left(X_{\sigma}^{s, x}\right)\right) d \sigma\right\}\right. \\
& \left.\cdot\left(\nabla_{x} \psi\left(\tau, X_{\tau}^{s, x}, Y_{\tau}^{n, s, x}, Z_{\tau}^{n, s, x}\right) \nabla_{x} X_{t}^{s, x}-n \dot{\gamma}\left(Y_{\tau}^{n, s, x}-h\left(X_{\tau}^{s, x}\right)\right) \nabla_{x} X_{\tau}^{s, x}\right) d \tau\right] \\
& +\mathbb{E}\left[\mathcal{E}_{T} \exp \left\{\int_{s}^{T} \nabla_{y} \psi\left(t, X_{\sigma}^{s, x}, Y_{\sigma}^{n, s, x}, Z_{\sigma}^{n, s, x}\right)+n \dot{\gamma}\left(Y_{\sigma}^{n, s, x}-h\left(X_{\sigma}^{s, x}\right)\right) d \sigma\right\} \nabla \phi\left(X_{T}^{s, x}\right) \nabla_{x} X_{T}^{s, x}\right] .
\end{aligned}
$$

Taking into account that $\nabla_{y} \psi$ is bounded by Hypothesis 2.7 and that $\dot{\gamma} \leq 0$ :

$$
\begin{aligned}
& \left|\nabla_{x} Y_{s}^{n, s, x}\right| \\
& \leq c \mathbb{E}\left[\mathcal{E}_{T}\left(\left|\nabla \phi\left(X_{T}^{s, x}\right) \nabla_{x} X_{T}^{s, x}\right|+\left|\int_{s}^{T} \nabla_{x} \psi\left(\tau, X_{\tau}^{s, x}, Y_{\tau}^{n, s, x}, Z_{\tau}^{n, s, x}\right) \nabla_{x} X_{\tau}^{s, x} d \tau\right|\right)\right] \\
& +c \mathbb{E}\left[\mathcal{E}_{T}\left|\int_{s}^{T} \exp \left\{\int_{s}^{\tau} n \dot{\gamma}\left(Y_{\sigma}^{n, s, x}-h\left(X_{\sigma}^{s, x}\right)\right) d \sigma\right\}\left(-n \dot{\gamma}\left(Y_{\tau}^{n, s, x}-h\left(X_{\tau}^{s, x}\right)\right)\right) \nabla h\left(X_{\tau}^{s, x}\right) \nabla_{x} X_{\tau}^{s, x} d \tau\right|\right] \\
& =I+I I .
\end{aligned}
$$

We start by estimating I. Here and in the following we again denote by $c$ a constant whose values can vary from line to line but does not depend neither on $n$ nor on $x$.

$$
I \leq c \mathbb{E}\left[\mathcal{E}_{T}\left|\nabla \phi\left(X_{T}^{s, x}\right) \nabla_{x} X_{T}^{s, x}\right|\right]+c \mathbb{E}\left[\mathcal{E}_{T} \int_{s}^{T}\left|\nabla_{x} \psi\left(\tau, X_{\tau}^{s, x}, Y_{\tau}^{n, s, x}, Z_{\tau}^{n, s, x}\right) \nabla_{x} X_{\tau}^{s, x}\right| d \tau\right] .
$$

Taking into account that $\mathbb{E} \mathcal{E}_{T}^{p} \leq c$, by Hölder inequality, with $p, q, r$ conjugate exponents $p>$ $1,1<q<2, q m>2$, (where $m$ is the same as in Hypothesis 2.7) we get:

$$
\mathbb{E}\left[\mathcal{E}_{T}\left|\nabla \phi\left(X_{T}^{s, x}\right) \nabla_{x} X_{T}^{s, x}\right|\right] \leq c\left(\mathbb{E}\left[\left|\nabla \phi\left(X_{T}^{s, x}\right)\right|^{q}\right]\right)^{1 / q}\left(\mathbb{E}\left[\left|\nabla_{x} X_{T}^{s, x}\right|^{r}\right]\right)^{1 / r} \leq c\left(1+|x|^{m}\right),
$$

where we have used the estimate on $\nabla_{x} X_{T}^{s, x}$ stated in [10], Proposition 3.3.

In a similar way we can estimate (for $q>2$ ):

$$
\begin{aligned}
& \mathbb{E}\left[\mathcal{E}_{T} \int_{s}^{T}\left|\nabla_{x} \psi\left(\tau, X_{\tau}^{s, x}, Y_{\tau}^{n, s, x}, Z_{\tau}^{n, s, x}\right) \nabla_{x} X_{\tau}^{s, x}\right| d \tau\right] \\
& \leq c \mathbb{E}\left[\mathcal{E}_{T} \int_{s}^{T}\left(1+\left|X_{\tau}^{s, x}\right|^{m}+\left|Y_{\tau}^{n, s, x}\right|^{m}\right)\left|Z_{\tau}^{n, s, x}\right|\left(1+\left|\nabla_{x} X_{\tau}^{s, x}\right|\right) d \tau\right] \\
& \leq c\left(\mathbb{E}\left[\left(1+\sup _{\tau \in[s, T]}\left|X_{\tau}^{s, x}\right|^{m q}+\sup _{\tau \in[s, T]}\left|Y_{\tau}^{n, s, x}\right|^{m q}\right)\left(1+\sup _{\tau \in[s, T]}\left|\nabla_{x} X_{\tau}^{s, x}\right|^{q}\right)\left(\int_{s}^{T}\left|Z_{\tau}^{n, s, x}\right| d \tau\right)^{q}\right]\right)^{1 / q} \\
& \leq c\left(\mathbb{E}\left[1+\sup _{\tau \in[s, T]}\left|X_{\tau}^{s, x}\right|^{2 m q}+\sup _{\tau \in[s, T]}\left|Y_{\tau}^{n, s, x}\right|^{2 m q}\right]\right)^{1 / 2 q}\left(\mathbb{E}\left[\int_{s}^{T}\left|Z_{\tau}^{n, s, x}\right| d \tau\right]^{2 q}\right)^{1 / 2 q} \\
& \leq c\left(1+|x|^{m(m+1)}\right) .
\end{aligned}
$$

where we have used estimates (2.20) and Proposition 2.6,

As far as $I I$ is concerned, let $p, q$ and $\bar{p}, \bar{q}$ be two pairs of conjugate exponents, and let

$$
l(\tau):=-n \dot{\gamma}\left(Y_{\tau}^{n, s, x}-h\left(X_{\tau}^{s, x}\right)\right) \geq 0, \tau \in[s, T]
$$


Then:

$$
\begin{aligned}
& \mathbb{E}\left[\mathcal{E}_{T}\left|\int_{s}^{T} \exp \left(-\int_{s}^{\tau} l_{\sigma} d \sigma\right) l_{\tau} \nabla h\left(X_{\tau}^{s, x}\right) \nabla_{x} X_{\tau}^{s, x} d \tau\right|\right] \\
& \leq c\left(\mathbb{E}\left[\left(1+\sup _{\tau \in[s, T]}\left|X_{\tau}^{s, x}\right|^{m}\right) \sup _{\tau \in[s, T]}\left|\nabla_{x} X_{\tau}^{s, x}\right| \int_{s}^{T} \exp \left(-\int_{s}^{\tau} l_{\sigma} d \sigma\right) l_{\tau} d \tau\right]^{q}\right)^{1 / q} \\
& \leq c\left(\mathbb{E}\left[\left(1+\sup _{\tau \in[s, T]}\left|X_{\tau}^{s, x}\right|^{m \bar{p} q}\right) \sup _{\tau \in[s, T]}\left|\nabla_{x} X_{\tau}^{s, x}\right|^{\bar{p} q}\right]\right)^{1 /(\bar{p} q)}\left(\mathbb{E}\left[\int_{s}^{T} \exp \left(-\int_{s}^{\tau} l_{\sigma} d \sigma\right) l_{\tau} d \tau\right]^{q \bar{q}}\right)^{1 /(q \bar{q})} \\
& \leq c\left(1+|x|^{m}\right)\left(\mathbb{E}\left[1-\exp \left(-\int_{s}^{\tau} l_{\sigma} d \sigma\right)\right]^{q \bar{q}}\right)^{1 /(q \bar{q})} \leq c\left(1+|x|^{m}\right),
\end{aligned}
$$

where, in the last inequality, we have used that:

$$
\int_{s}^{T} \exp \left\{-\int_{s}^{\tau} l(\sigma) d \sigma\right\} l(\tau) d \tau=1-\exp \left\{-\int_{s}^{T} l(\sigma) d \sigma\right\}
$$

So

$$
\left|\nabla_{x} Y_{s}^{n, s, x}\right| \leq c\left(1+|x|^{m(m+1)}\right)
$$

where $c$ may depend on $T$, on the coefficients $A, F, G, \psi, h, \phi$, but not on $n$. By (2.29) we get that, for any $x, y \in H$ :

$$
\left|Y_{s}^{n, s, x}-Y_{s}^{n, s, y}\right| \leq c|x-y|\left(1+|x|^{m(m+1)}+|y|^{m(m+1)}\right) .
$$

By letting $n \rightarrow \infty$ (see (2.5)) we get the desired Lipschitz continuity of $Y_{s}^{s, x}$, namely:

$$
\left|Y_{s}^{s, x}-Y_{s}^{s, y}\right| \leq c|x-y|\left(1+|x|^{m(m+1)}+|y|^{m(m+1)}\right), \quad \forall x, y \in H .
$$

We notice that in the equations (2.30) and (2.31) the constant $c$ depends on $\phi, h$ and $\psi$ only through the constants $L$ and $L^{\prime}$. We have now to remove the assumption of differentiability on the coefficient $\psi, h, \phi$ in the reflected BSDE. Since $\psi$ is Lipschitz continuous with respect to $y$ and $z$, and $\forall t, y, z \in[0, T] \times \mathbb{R} \times \Xi, \psi(t, \cdot, y, z), h, \phi$ are locally Lipschitz continuous with respect to $x$, then by taking their inf-sup convolution $\left(\psi_{k}, \phi_{k}, h_{k}\right)_{k \geq 1}$ (see e.g. [4] for the notion of inf-sup convolution, and [15] and [16] for the use of inf-sup convolutions in the Lipschitz and locally Lipschitz case) we obtain a sequence of differentiable functions that verify Hypothesis 2.7 with constants $L$ and $L^{\prime}$ independent on $k$ and that converge pointwise to $(\psi, \phi, h)$.

We denote by $\left(Y^{n, k, s, x}, Z^{n, k, s, x}, K^{n, k, s, x}\right)$ the solution of the penalized RBSDEs with regularized coefficients:

$$
\left\{\begin{array}{l}
-d Y_{t}^{n, k, s, x}=\psi_{k}\left(t, X_{t}^{s, x}, Y_{t}^{n, k, s, x}, Z_{t}^{n, k, s, x}\right) d t+n \gamma\left(Y_{t}^{n, k, s, x}-h_{k}\left(X_{t}^{s, x}\right)\right) d t-Z_{t}^{n, k, s, x} d W_{t} \\
Y_{T}^{n, k, s, x}=\phi_{k}\left(X_{T}^{s, x}\right)
\end{array}\right.
$$

By (2.30) and (2.31) we get that for any $x, y \in H$ :

$$
\left|Y_{s}^{n, k, s, x}-Y_{s}^{n, k, s, y}\right| \leq c|x-y|\left(1+|x|^{m(m+1)}+|y|^{m(m+1)}\right),
$$

where $c$ does not depend nor on $n$ neither on $k$. By standard results on BSDEs (see [11]) we know that

$$
\left(Y^{n, k, s, x}, Z^{n, k, s, x}\right) \rightarrow\left(Y^{n, s, x}, Z^{n, s, x}\right) \text { in } L_{\mathcal{P}}^{p}(\Omega, C([0, T])) \times L_{\mathcal{P}}^{p}(\Omega \times[0, T], \Xi),
$$


where $\left(Y^{n, s, x}, Z^{n, s, x}\right)$ is the solution of equation (2.25). In particular $Y_{s}^{n, k, s, x} \rightarrow Y_{s}^{n, s, x}$. Finally by (2.5) we have $Y^{n, s, x} \uparrow Y^{s, x}$ (the fact that here the smooth and strictly monotone function $\gamma$ replaces the negative part $(\cdot)^{-}$is inessential). The claim then follows by $(2.33)$.

Remark 2.11 Notice that if $h$ and $\phi$ are bounded and Lipschitz, if for every $s \in[0, T]$, $\sup _{x \in H}|\psi(s, x, 0,0)|<\infty$ and, as a function of $x, \psi$ is Lipschitz uniformly with respect to the other variables (that is Hypothesis 2.7, point 3 holds true with $m=0$ ) then, by repeating the same arguments as in Proposition 2.8, we can prove that the processes $Y^{s, x}, Z^{s, x}$ are bounded with respect to $x$, that is:

$$
\mathbb{E} \sup _{t \in[s, T]}\left|Y_{t}^{s, x}\right|^{p}+\mathbb{E}\left(\int_{0}^{T}\left|Z_{t}^{s, x}\right|^{2} d t\right)^{p / 2}<C .
$$

\section{Obstacle problem for a semilinear parabolic PDE: solution via RBSDEs}

In this section we consider an obstacle problem for a semilinear PDE in an infinite dimensional Hilbert space $H$ and we solve it in a suitable sense by means of reflected BSDEs. We will deal with the following obstacle problem, formally written:

$$
\begin{cases}\min \left(u(t, x)-h(x),-\frac{\partial u}{\partial t}(t, x)-\mathcal{L}_{t} u(t, x)-\psi(t, x, u(t, x),\right. & \nabla u(t, x) G(t, x)))=0 \\ u(T, x)=\phi(x), & t \in[0, T], x \in H\end{cases}
$$

where the directional generalized gradient $\nabla u(t, x) G(t, x)$ will be defined below (also see [13]). Moreover $\left(\mathcal{L}_{t}\right)_{t \in[0, T]}$ is the infinitesimal generator of the Markov process generated by equation (2.18), namely, for a sufficiently smooth function $f: H \rightarrow \mathbb{R}, \mathcal{L}_{t}$, is defined by:

$$
\mathcal{L}_{t} f(x)=\frac{1}{2} \operatorname{Trace}\left(G(t, x) G^{*}(t, x) \nabla^{2} f(x)\right)+\langle A x, \nabla f(x)\rangle_{H}+\langle F(t, x), \nabla f(x)\rangle_{H} .
$$

More precisely if $X^{s, x}, 0, \leq s \leq t \leq T$ is the mild solution to equation (2.18) and we denote by $P_{s, t}$ the operator

$$
P_{s, t}[f](x)=\mathbb{E} f\left(X_{t}^{s, x}\right), \quad f \in C_{b}(H)
$$

then $\mathcal{L}_{t}$ is (at least formally) the generator of the transition semigroup $\left(P_{s, t}\right)_{0, \leq s \leq t \leq T}$.

The above leads us to consider solutions of the obstacle problem (3.1) in mild sense, as we are going to state in the next sections

\subsection{The generalized directional gradient}

We observe that, under our assumptions we can not expect that the value function of an optimal stopping problem is differentiable. For instance, consider the following trivial deterministic state equation without control:

$$
\left\{\begin{array}{l}
d X_{t}^{s, x}=0 \\
X_{s}^{s, x}=x \in \mathbb{R}
\end{array}\right.
$$

and the following stopping problem:

$$
J(s, x, \tau)=\phi\left(X_{T}^{s, x}\right) \chi_{\{\tau=T\}}+h\left(\tau, X_{\tau}^{s, x}\right) \chi_{\{\tau<T\}},
$$


It is evident that the value function is given by:

$$
v(x)=\sup _{\tau}\left(\phi\left(X_{T}^{s, x}\right) \chi_{\{\tau=T\}}+h\left(\tau, X_{\tau}^{s, x}\right) \chi_{\{\tau<T\}}\right)=\max (\phi(x), h(x)),
$$

and that, even if the data are differentiable, $v$ may fail to be differentiable (if $\phi(x)=x$ and $h(x)=-x$ then $v(x)=|x|)$.

Notice that in the above example and statement we heavily rely on the degeneracy of the noise. The issue of differentiability of $u$ when noise is non degenerate is very interesting but falls out of the scope of the present work.

To handle the lack of regularity of $u$ the classical derivative $\nabla u$ must not appear in the precise formulation of the problem. Indeed it will be replaced by the notion of generalized gradient, whose definition is given below.

Theorem 3.1 Assume that Hypothesis 2.5 holds and that $u:[0, T] \times H \rightarrow \mathbb{R}$ is a Borel measurable function satisfying, for some $r>0$

$$
\left|u(t, x)-u\left(t, x^{\prime}\right)\right| \leq c\left(1+|x|^{r}+\left|x^{\prime}\right|^{r}\right)\left|x-x^{\prime}\right| .
$$

Then there exists a Borel measurable function $\zeta:[0, T] \times H \rightarrow \Xi^{*}$ with the following properties.

(i) For every $s \in[0, T], x \in H$ and $p \in[2, \infty)$,

$$
\mathbb{E} \int_{s}^{T}\left|\zeta\left(\tau, X_{\tau}^{t, x}\right)\right|^{p} d \tau<+\infty
$$

(ii) For any $\xi \in \Xi$ denote by $W^{\xi}=\left(W_{t}^{\xi}\right)_{t \geq 0}$ the projection: $W_{t}^{\xi}:=\left\langle W_{t}, \xi\right\rangle$. Then, for any $x \in H$ and $0 \leq s \leq T^{\prime}<T$, the processes $\left\{u\left(t, X_{t}^{s, x}\right), t \in[s, T]\right\}$ and $W^{\xi}$ admit a joint quadratic variation on the interval $\left[s, T^{\prime}\right]$ and

$$
\left\langle u\left(\cdot, X^{s, x}\right), W^{\xi}\right\rangle_{\left[s, T^{\prime}\right]}=\int_{s}^{T^{\prime}} \zeta\left(t, X_{t}^{s, x}\right) \xi d t, \quad \mathbb{P}-\text { a.s.. }
$$

(iii) There exists a Borel measurable function $\rho:[0, T] \times H \rightarrow H^{*}$ such that for all $t \in[s, T]$ and all $x \in H$

$$
\zeta\left(t, X_{t}^{s, x}\right)=\rho\left(t, X_{t}^{s, x}\right) G\left(t, X_{t}^{s, x}\right) \quad \mathbb{P} \text {-a.s. for a.e. } t \in[s, T] .
$$

Proof. The proof is given in [13], section 4. In that paper it is also noticed, see remark 3.1, that uniqueness can be stated in the following sense: if $\hat{\zeta}$ is another function with the stated properties then for $0 \leq s \leq t \leq T$ and $x \in H$ we have $\zeta\left(t, X_{t}^{s, x}\right)=\hat{\zeta}\left(t, X_{t}^{s, x}\right), \mathbb{P}-$ a.s. for a.e. $t \in[s, T]$.

Definition 3.1 Let $u:[0, T] \times H \rightarrow \mathbb{R}$ be a Borel measurable function satisfying (3.2). The family of all measurable functions $\zeta:[0, T] \times H \rightarrow \Xi^{*}$ satisfying properties (i) and (ii) in Theorem 3.1 will be called the generalized directional gradient of $u$ and denoted by $\widetilde{\nabla}^{G} u$.

\subsection{Mild solutions of the obstacle problem in the sense of the generalized directional gradient}

We are now in the position to give the precise definition of supersolution for the problem (3.1). 
Definition 3.2 We say that a Borel measurable function $\bar{u}:[0, T] \times H \rightarrow \mathbb{R}$ is a mild supersolution of the obstacle problem (3.1) in the sense of the generalized directional gradient if the following holds:

1. for some $C>0, r \geq 0$ and for every $s \in[0, T], x, x^{\prime} \in H$

$$
\left|\bar{u}(s, x)-\bar{u}\left(s, x^{\prime}\right)\right| \leq C\left|x-x^{\prime}\right|\left(1+|x|+\left|x^{\prime}\right|\right)^{r}, \quad|u(s, 0)| \leq C ;
$$

2. for every $s \in[0, T], x \in H$,

$$
\bar{u}(s, x) \geq h(x)
$$

3. for all $0 \leq s \leq t \leq T$ and $x \in H$

$$
\bar{u}(s, x) \geq P_{s, t}[u(t, \cdot)](x)+\int_{s}^{t} P_{s, \tau}[\psi(\tau, \cdot, \bar{u}(\tau, \cdot), \zeta(\tau, \cdot))](x) d \tau,
$$

where $\zeta$ is an arbitrary element of the generalized gradient $\widetilde{\nabla}^{G} \bar{u}$;

4. $\bar{u}(T, \cdot)=\phi$.

Remark 3.2 We notice that the notion of supersolution stated above is related to the inequality in formula (3.4) and does not refer to the definition of supersolution in viscosity sense (see, for instance [9]). In this paper the issue of uniqueness of the solution to the obstacle problem is bypassed by constructing a minimal supersolution but it remains essentially open. We just recall that viscosity theory (at least in its classical formulation) will probably not be helpful since it requires strong assumptions to be applied to infinite dimensional HJB equations even when there is no obstacle.

We are now ready to state the main result of this paper.

Theorem 3.3 Assume that Hypotheses 2.5 and 2.7 hold true. Let us define

$$
u(s, x)=Y_{s}^{s, x},
$$

where $\left(Y^{s, x}, Z^{s, x}\right)$ is solution to the reflected BSDE in (2.15). Then $u$ is a mild supersolution in the sense of the generalized directional gradient for the obstacle problem (3.1).

Moreover $u$ is minimal in the sense that given any $\bar{u}$, supersolution of (3.1) in the sense of Definition 3.2, it holds $u(s, x) \leq \bar{u}(s, x)$, and $s \in[0, T], x \in H$

Finally, if in addition $\sup _{s \in[0, T], x \in H}|\psi(s, x, 0,0)|<\infty$ and $\phi$ and $h$ are bounded then $u$ is also bounded.

Proof. If we set $u(s, x):=Y_{s}^{s, x}$ then, by Theorem 2.10, $u$ has the regularity required in definition 3.2 , point 1 , moreover points 2 and 4 immediately follow since $Y$ the is solution to the RBSDE in $(2.15)$.

As far as point 3 of definition 3.2 is concerned, by (2.15) we get

$$
u(s, x)=Y_{t}^{s, x}+\int_{s}^{t} \psi\left(\tau, X_{\tau}^{s, x}, Y_{\tau}^{s, x}, Z_{\tau}^{s, x}\right) d \tau+K_{t}^{s, x}-K_{s}^{s, x}-\int_{s}^{t} Z_{\tau}^{s, x} d W_{\tau},
$$

Fixed $\xi \in \Xi$, let us consider the joint quadratic variation of both sides of (3.6) with $W^{\xi}$. Proposition 2.1 in [13] and Theorem 3.1 yield that $\widetilde{\nabla}^{G} u$ exists and letting $\zeta \in \widetilde{\nabla}^{G} u$, we have

$$
\left\langle u\left(\cdot, X^{s, x}\right), W_{\cdot}^{\xi}\right\rangle_{[s, t]}=\int_{s}^{t} \zeta\left(\sigma, X_{\sigma}^{s, x}\right) \xi d \sigma
$$


On the other hand by the Markov property stated in Remark 2.9

$$
u\left(t, X_{t}^{s, x}\right)=Y_{t}^{t, X_{t}^{s, x}}=Y_{t}^{s, x}
$$

and by (2.15) we deduce:

$$
\left\langle Y^{s, x}, W \cdot\right\rangle_{[s, t]}=\int_{s}^{t} Z_{\sigma}^{s, x} \xi d \sigma .
$$

So, by these two expression of the joint quadratic variation of $u\left(\cdot, X^{s, x}\right)$ and $W^{\xi}$ we get

$$
\int_{s}^{t} \zeta\left(\sigma, X_{\sigma}^{s, x}\right) \xi d \sigma=\int_{s}^{t} Z_{\sigma}^{s, x} \xi d \sigma
$$

$\mathbb{P}$-a.s. Since both sides of (3.7) are continuous with respect to $t$, it follows that, $\mathbb{P}$-a.s., they coincide for all $t \in[s, T]$. This implies that $\zeta\left(\sigma, X_{\sigma}^{s, x}\right)=Z_{\sigma}^{s, x}, \mathbb{P}$-a.s. for a.e. $\sigma \in[s, T]$. Therefore equation (3.6) can be rewritten as

$$
u(s, x)=Y_{t}^{s, x}+\int_{s}^{t} \psi\left(\tau, X_{\tau}^{s, x}, u\left(\tau, X_{\tau}^{s, x}\right), \zeta\left(\tau, X_{\tau}^{s, x}\right)\right) d \tau+K_{t}^{s, x}-K_{s}^{s, x}-\int_{s}^{t} Z_{\tau}^{s, x} d W_{\tau} .
$$

By taking the conditional expectation $\mathbb{E}^{\mathcal{F}_{s}}$, since $K$ is a nondecreasing we get:

$$
u(s, x) \geq Y_{t}^{s, x}+\int_{s}^{t} P_{s, \tau}[\psi(\tau, \cdot, u(\tau, \cdot), \zeta(\tau, \cdot))](x) d \tau .
$$

We have shown that $u$ is a mild supersolution along Definition 3.2.

Now we have to prove that $u$ is the minimal supersolution. Let $\bar{u}$ be any supersolution and let us define $\bar{Y}_{t}^{s, x}=\bar{u}\left(t, X_{t}^{s, x}\right)$. Then for every $\sigma \in[s, t]$, with $0 \leq s \leq t$, by point 3 of definition 3.2 , having replaced $x$ with $X_{\sigma}^{s, x}$ which is $\mathcal{F}_{\sigma}$-measurable,

$$
\bar{u}\left(\sigma, X_{\sigma}^{s, x}\right) \geq \mathbb{E}^{\mathcal{F}_{\sigma}} \bar{u}\left(t, X_{t}^{\sigma, X_{\sigma}^{s, x}}\right)+\mathbb{E}^{\mathcal{F}_{\sigma}} \int_{\sigma}^{t} \psi\left(\tau, X_{\tau}^{\sigma, X_{\sigma}^{s, x}}, \bar{Y}_{\tau}^{\sigma, X_{\sigma}^{s, x}}, \bar{\zeta}\left(\tau, X_{\tau}^{\sigma, X_{\sigma}^{s, x}}\right) d \tau .\right.
$$

So it turns out that

$$
\left(L_{\sigma}^{s, x}\right)_{\sigma \in[s, T]}:=\left(-\bar{u}\left(\sigma, X_{\sigma}^{s, x}\right)-\int_{s}^{\sigma} \psi\left(\tau, X_{\tau}^{s, x}, \bar{Y}_{\tau}^{s, x}, \bar{\zeta}\left(\tau, X_{\tau}^{s, x}\right)\right) d \tau\right)_{\sigma \in[s, T]}
$$

is a submartingale. By Hypothesis 2.7 on $\psi$; by the growth property of $u$ as required in definition 3.2 , point 1 ; by relation 3.3 and finally by Proposition 2.6 we get that $L^{s, x}$ is a uniformly integrable submartingale, so it is of class (D) and the Doob-Meyer decomposition can be applied, see e.g. Definition 4.8 and Theorem 4.10 in Chapter 1 of [8]. Therefore $L^{s, x}$ can be decomposed into:

$$
L_{\sigma}^{s, x}=\bar{M}_{\sigma}^{s, x}+\bar{K}_{\sigma}^{s, x},
$$

where $\bar{K}^{s, x}$ is an integrable nondecreasing process such that $\bar{K}_{s}^{s, x}=0$, and $\bar{M}^{s, x}$ is a uniformly integrable martingale. Moreover (see [5], Chapter VII relation (15.1)) since

$$
\mathbb{E} \sup _{\sigma \in[s, T]}\left|L_{\sigma}^{s, x}\right|^{2}<\infty
$$

we have

$$
\bar{K}_{T}^{s, x} \in L^{2}(\Omega) .
$$


Notice that $\left(\bar{M}_{t}^{s, x}\right)_{t \in[s, T]}$ is a martingale with respect to the complete filtration generated by the Wiener process, thus, by the martingale representation theorem, see again [8] and [3], there exists a process $\bar{Z} \in L_{\mathcal{P}}^{2}\left(\Omega \times[s, T] ; L_{2}(\Xi, \mathbb{R})\right)$ such that

$$
\bar{M}_{\sigma}^{s, x}=-\left[u(s, x)+\int_{s}^{\sigma} \bar{Z}_{\tau}^{s, x} d W_{\tau}\right] .
$$

The above relation together with the definition of $L^{s, x}$ yield for $\forall \sigma \in[s, T]$

$$
u(s, x)=\bar{u}\left(\sigma, X_{\sigma}^{s, x}\right)+\int_{s}^{\sigma} \psi\left(\tau, X_{\tau}^{s, x}, \bar{Y}_{\tau}^{s, x}, \bar{\zeta}\left(\tau, X_{\tau}^{s, x}\right) d \tau+\bar{K}_{\sigma}^{s, x}-\bar{K}_{s}^{s, x}-\int_{s}^{t} \bar{Z}_{\tau}^{s, x} d W_{\tau},\right.
$$

that is, $\forall 0 \leq s \leq t \leq T$ :

$$
\bar{Y}_{t}^{s, x}=\bar{Y}_{T}^{s, x}+\int_{t}^{T} \psi\left(\tau, X_{\tau}^{s, x}, \bar{Y}_{\tau}^{s, x}, \bar{\zeta}\left(\tau, X_{\tau}^{s, x}\right)\right) d \tau+\bar{K}_{t}^{s, x}-\bar{K}_{s}^{s, x}-\int_{s}^{t} \bar{Z}_{\tau}^{s, x} d W_{\tau} .
$$

Finally we have to identify $\zeta\left(\tau, X_{\tau}^{s, x}\right)$ with $\bar{Z}_{\tau}^{s, x}, \mathbb{P}$-a.s. for a.e. $\tau \in[s, T]$. To this aim, for $\xi \in \Xi$, let us consider the joint quadratic variation of both sides of (3.11) with $W^{\xi}$. Notice that the finite variation term $K$ does not give any contribution. So Proposition 2.1 in [13] and Theorem 3.1 yield, for $s \leq \sigma<T$ and $\zeta \in \widetilde{\nabla}^{G} u$,

$$
\int_{s}^{\sigma} \zeta\left(\tau, X_{\tau}^{s, x}\right) \xi d \tau=\int_{s}^{\sigma} \bar{Z}_{\tau}^{s, x} \xi d \tau
$$

$\mathbb{P}$-a.s.. Since both sides of (3.13) are continuous with respect to $\sigma$, it follows that, $\mathbb{P}$-a.s., they coincide for all $\sigma \in[s, T]$. This implies that $\zeta\left(\sigma, X_{\sigma}^{s, x}\right)=\bar{Z}_{\sigma}^{s, x}$, P-a.s. for a.e. $\sigma \in[s, T]$. So we get that, by defining: $\bar{Y}_{t}^{s, x}:=\bar{u}\left(t, X_{t}^{s, x}\right), t \in[s, T]$ the couple of processes $\left(\bar{Y}^{s, x}, \bar{Z}^{s, x}\right)$ verifies:

$$
\left\{\begin{array}{l}
-d \bar{Y}_{t}^{s, x}=\psi\left(t, X_{t}^{s, x}, \bar{Y}_{t}^{s, x}, \bar{Z}_{t}^{s, x}\right) d t+d \bar{K}_{t}^{s, x}-\bar{Z}_{t}^{s, x} d W_{t}, \quad t \in[s, T] \\
\bar{Y}_{T}=\phi\left(X_{T}^{s, x}\right) \\
\bar{Y}_{t}^{s, x} \geq h\left(X_{t}^{s, x}\right)
\end{array}\right.
$$

Now we have to compare $\bar{Y}^{s, x}$ with $Y^{s, x}$. To this aim, extending a procedure used in [2], we compare $\bar{Y}^{s, x}$ with the penalized solution $Y^{n, s, x}$ of equation (2.21). where we have taken into account that $y+(y-h)^{-}=y \vee h$.

Applying Itô formula to the process $e^{n(T-t)} Y_{t}^{n, s, x}$ we get:

$$
\left\{\begin{array}{c}
-d e^{n(T-t)} Y_{t}^{n, s, x}=e^{n(T-t)} \psi\left(t, X_{t}^{s, x}, Y_{t}^{n, s, x}, Z_{t}^{n, s, x}\right) d t+n e^{n(T-t)} Y_{t}^{n, s, x} \vee h\left(X_{t}^{s, x}\right) d t \\
-e^{n(T-t)} Z_{t}^{n, s, x} d W_{t}, \quad t \in[s, T], \\
Y_{T}^{n, s, x}=\phi\left(X_{T}^{s, x}\right) .
\end{array}\right.
$$

Applying Itô formula to the process $e^{n(T-t)} \bar{Y}_{t}^{s, x}$ we get

$$
\left\{\begin{array}{l}
-d e^{n(T-t)} \bar{Y}_{t}^{s, x}=n e^{n(T-t)} \bar{Y}_{t}^{s, x} d t+e^{n(T-t)} \psi\left(t, X_{t}^{s, x}, \bar{Y}_{t}^{s, x}, \bar{Z}_{t}^{s, x}\right) d t+e^{n(T-t)} d \bar{K}_{t}^{s, x} \\
-e^{n(T-t)} \bar{Z}_{t}^{s, x} d W_{t}, \quad t \in[s, T], \\
\bar{Y}_{T}^{s, x}=\phi\left(X_{T}^{s, x}\right) .
\end{array}\right.
$$

Notice that in (3.16) we can replace $\bar{Y}_{t}^{s, x} \vee h\left(X_{t}^{s, x}\right)$ by $\bar{Y}_{t}^{s, x}$ (recall that $\bar{u} \geq h$ since $\bar{u}$ is a supersolution to the obstacle problem (3.1)). Assume for a moment the following Lemma. 
Lemma 3.4 Let $f^{i}: \Omega \times[0, T] \times \mathbb{R} \times \Xi \rightarrow \mathbb{R}, i=1,2$ satisfy hypothesis 2.2 with $p=2$, fix $\xi \in L_{\mathcal{F}_{T}}^{2}(\Omega)$ let $K$ be a progressively measurable nondecreasing processes with $\mathbb{E} K_{T}^{2}<\infty$. If $\left(Y^{1}, Z^{1}\right)$ and $\left(Y^{2}, Z^{2}\right)$ with $Y^{i} \in L_{\mathcal{P}}^{2}(\Omega, C([0, T]))$ and $Z^{i} \in L_{\mathcal{P}}^{2}(\Omega \times[0, T], \Xi), i=1,2$, are the solutions to the following equations of backward type:

$$
\begin{gathered}
\left\{\begin{array}{l}
-d Y_{t}^{1}=f^{1}\left(t, Y_{t}^{1}, Z_{t}^{1}\right) d t+d K_{t}-Z_{t}^{1} d W_{t}, \quad t \in[0, T], \\
Y_{T}^{1}=\xi,
\end{array}\right. \\
\left\{\begin{array}{l}
-d Y_{t}^{2}=f^{2}\left(t, Y_{t}^{2}, Z_{t}^{2}\right) d t-Z_{t}^{2} d W_{t}, \quad t \in[0, T], \\
Y_{T}^{2}=\xi .
\end{array}\right.
\end{gathered}
$$

and

$$
(\delta f)_{t}:=f^{1}\left(t, Y_{t}^{2}, Z_{t}^{2}\right)-f^{2}\left(t, Y_{t}^{2}, Z_{t}^{2}\right) \geq 0, d \mathbb{P} \times d t \quad a . s,
$$

then we have that $Y_{t}^{1} \geq Y_{t}^{2}, \mathbb{P}$-almost surely for any $t \in[0, T]$.

By applying Lemma 3.4 to the BSDEs (3.15) and (3.16) we get a comparison for the processes $\left(e^{n(T-t)} Y_{t}^{n, s, x}\right)_{t \in[s, T]}$ and $\left(e^{n(T-t)} \bar{Y}_{t}^{s, x}\right)_{t \in[s, T]}$, namely we get

$$
e^{n(T-t)} \bar{Y}_{t}^{s, x} \geq e^{n(T-t)} Y_{t}^{n, s, x}
$$

almost surely and for any time $t$, and consequently

$$
\bar{Y}_{t}^{s, x} \geq Y_{t}^{n, s, x} .
$$

Now we let $n \rightarrow \infty$ : by [6], section $6, Y_{t}^{n, s, x} \uparrow Y_{t}^{s, x}$ for any $s \leq t \leq T$ and $\mathbb{P}$-a.s.. So taking $s=t$ in (3.21) we finally get

$$
\bar{u}(s, x) \geq u(s, x) .
$$

So the minimality of $u$ is proved.

The other properties required by Definition 3.2 follow by estimates (2.20), which passes to the limit as $n \rightarrow \infty$.

In order to complete the proof of Theorem 3.3, we have to prove Lemma 3.4.

Proof of Lemma 3.4. We adapt the proof of the classical comparison theorem for BSDEs given in [7], Theorem 2.2, to the equations (3.17) and (3.18). Let:

$$
\Delta_{y} f_{t}^{1}=\frac{f^{1}\left(t, Y_{t}^{1}, Z_{t}^{1}\right)-f^{1}\left(t, Y_{t}^{2}, Z_{t}^{1}\right)}{Y_{t}^{1}-Y_{t}^{2}} \quad \text { if } Y_{t}^{1}-Y_{t}^{2} \neq 0, \quad \Delta_{y} f_{t}^{1}=0 \text { otherwise, }
$$

$\Delta_{z} f_{t}^{1}=\frac{f^{1}\left(t, Y_{t}^{2}, Z_{t}^{1}\right)-f^{1}\left(t, Y_{t}^{2}, Z_{t}^{2}\right)}{\left|Z_{t}^{1}-Z_{t}^{2}\right|^{2}}\left(Z_{t}^{1}-Z_{t}^{2}\right) \quad$ if $Z_{t}^{1}-Z_{t}^{2} \neq 0, \quad \Delta_{z} f_{t}^{1}=0$ otherwise.

Moreover let $(\delta f)$ be defined as in (3.19), and $(\delta Y)_{t}:=Y_{t}^{1}-Y_{t}^{2},(\delta Z)_{t}:=Z_{t}^{1}-Z_{t}^{2}$.

It holds

$$
\left\{\begin{array}{l}
-d(\delta Y)_{t}=\Delta_{y} f_{t}^{1}(\delta Y)_{t} d t+\left(\Delta_{z} f_{t}^{1}\right)^{*}(\delta Z)_{t} d t+(\delta f)_{t} d t+d K_{t}-(\delta Z)_{t} d W_{t}, \quad t \in[0, T] \\
(\delta Y)_{T}=0 .
\end{array}\right.
$$

We notice that $\Delta_{y} f_{t}^{1}$ and $\Delta_{z} f_{t}^{1}$ are bounded and that $(\delta f) \in L_{\mathcal{P}}^{2}(\Omega \times[0, T], \mathbb{R})$.

Multiplying $(\delta Y)_{t}$ by $\exp \left(\int_{0}^{t} \Delta_{y} f_{\tau}^{1} d \tau\right)$ and then applying Girsanov theorem we obtain: 


$$
(\delta Y)_{t}=\mathbb{E}\left(\rho_{t, T}\left[\int_{t}^{T} \exp \left(\int_{t}^{s} \Delta_{y} f_{\tau}^{1} d \tau\right) d K_{s}+\int_{t}^{T} \exp \left(\int_{t}^{s} \Delta_{y} f_{\tau}^{1} d \tau\right)(\delta f)_{s} d s\right] \mid \mathcal{F}_{t}\right)
$$

where $\rho_{t, T}$ is the Girsanov density:

$$
\rho_{t, T}=\exp \left(\int_{t}^{T}\left(\Delta_{z} f_{s}^{1}\right)^{*} d W_{s}-\frac{1}{2} \int_{t}^{T}\left|\Delta_{z} f_{s}^{1}\right|^{2} d s\right) .
$$

The claim follows from (3.24) since $\left(K_{t}\right)$ is a non decreasing process and $(\delta f)_{t} \geq 0, \mathbb{P}$ a.s. for a.e. $t \in[0, T]$.

\section{The Optimal Control-Stopping problem}

We apply our results to an optimanl control and stopping problem. We start by specifying the setting. An Admissible System will be a set

$$
\mathcal{S}=\left(\Omega^{\mathcal{S}}, \mathcal{F}^{\mathcal{S}},\left(\mathcal{F}_{t}^{\mathcal{S}}\right)_{t \geq 0}, \mathbb{P}^{\mathcal{S}},\left(W_{t}^{\mathcal{S}}\right)_{t \geq 0}\right)
$$

where $\left(\Omega^{\mathcal{S}}, \mathcal{F}^{\mathcal{S}},\left(\mathcal{F}_{t}^{\mathcal{S}}\right)_{t \geq 0}, \mathbb{P}^{\mathcal{S}}\right)$, is a complete probability space endowed with a filtration satisfying the usual assumptions and $\left.\left(W_{t}^{\mathcal{S}}\right)_{t \geq 0}\right)$ is a $\left(\mathcal{F}^{\mathcal{S}}\right)$ - cylindrical Wiener process in $\Xi$. Fixed a closed subset $U$ of a normed space $U_{0}$ an admissible control in the setting $\mathcal{S}$ is any $\left(\mathcal{F}_{t}^{\mathcal{S}}\right)$-predictable process $\alpha: \Omega^{\mathcal{S}} \times[0, T] \rightarrow U$. The set of all admissible controls will be denoted by $\mathcal{U}^{\mathcal{S}}$.

We fix a function $R: H \times U \rightarrow \Xi$ bounded, continuous such that:

$$
\left|R(\alpha, x)-R\left(\alpha, x^{\prime}\right)\right| \leq\left|x-x^{\prime}\right| \quad \forall \alpha \in U, x, x^{\prime} \in H
$$

Given an admissible setting $\mathcal{S}$, an admissible control $\alpha \in \mathcal{U}^{\mathcal{S}}$, an initial time $s \in[0, T]$ and an initial datum $x \in H$, by $X^{\alpha, s, x}$ we denote the solution to the following stochastic differential equation in $H$ (notice that $X^{\alpha, s, x}$ depends on $\mathcal{S}$ but for notational simplicity we do not indicate it)

$$
\left\{\begin{array}{l}
d X_{t}^{\alpha, s, x}=A X_{t}^{\alpha, s, x} d t+F\left(t, X_{t}^{\alpha, s, x}\right) d t+G\left(t, X_{t}^{\alpha, s, x}\right)\left(R\left(X_{t}^{\alpha, s, x}, \alpha_{t}\right) d t+d W_{t}^{\mathcal{S}}\right), \quad t \in[s, T] \\
X_{s}^{\alpha, s, x}=x \in H .
\end{array}\right.
$$

Moreover given $l:[0, T] \times H \times U_{0} \rightarrow \mathbb{R}$ we introduce the cost functional:

$$
J(s, x, \tau, \alpha)=\mathbb{E} \int_{s}^{\tau} l\left(r, X_{r}^{\alpha, s, x}, \alpha_{r}\right) d r+\mathbb{E}\left[\phi\left(X_{T}^{\alpha, s, x}\right) \chi_{\{\tau=T\}}\right]+\mathbb{E}\left[h\left(\tau, X_{\tau}^{\alpha, s, x}\right) \chi_{\{\tau<T\}}\right],
$$

that we wish to maximize over all admissible control $\alpha \in \mathcal{U}^{\mathcal{S}}$ and over all $\left\{\mathcal{F}_{t}^{\mathcal{S}}\right\}_{t}$-stopping times $\tau$ satisfying $t \leq \tau \leq T$ (the mean value is computed with respect to $\mathbb{P}^{\mathcal{S}}$ ).

For $s \in[0, T], x \in H, z \in \Xi^{*}$ we define the Hamiltonian function in the usual way as

$$
\psi(s, x, z)=\sup _{\alpha \in U}\{z R(x, \alpha)+l(s, x, \alpha)\} .
$$

We notice that since $R$ is bounded $\psi$ is Lipschitz with respect to $z$. We will assume throughout this section that $A, F$ and $G$ verify Hypothesis 2.5 and that $\phi, \psi$ and $h$ verify Hypothesis 2.7. Moreover we assume that $|l(s, x, \alpha)| \leq c\left(1+|x|^{r}\right)$ for some $c, r>0$.

Under the above assumptions, fixed $s \in[0, T]$ and $x \in H$, for all $\alpha \in \mathcal{U}^{\mathcal{S}}$ there exists a unique mild solution $X^{\alpha, s, x}$ to equation (4.2). Moreover $X^{\alpha, s, x} \in L_{\mathcal{P}}^{p}(\Omega, C([s, T], H))$ for all 
$p \geq 1$, see [10]. Consequently $J(s, x, \tau, \alpha)$ is a well defined real number for all $\alpha \in \mathcal{U}^{\mathcal{S}}$ and all $\left\{\mathcal{F}_{t}^{\mathcal{S}}\right\}_{t}$-stopping time $\tau \leq T$.

By the Girsanov theorem, there exists a probability measure $\mathbb{P}^{\mathcal{S}, \alpha}$ such that the process

$$
W_{t}^{\mathcal{S}, \alpha}:=W_{t}^{\mathcal{S}}+\int_{s}^{t} R\left(X_{r}^{\alpha, s, x}, \alpha_{r}\right) d r \quad t \geq s
$$

is a cylindrical $\mathbb{P}^{\mathcal{S}, \alpha}$-Wiener process in $\Xi$. We denote by $\left(\mathcal{F}_{t}^{\mathcal{S}, \alpha}\right)_{t \geq s}$ its natural filtration, augmented in the usual way. $X^{\alpha, s, x}$ satisfies the following equation:

$$
\left\{\begin{array}{l}
d X_{t}^{\alpha, s, x}=A X_{t}^{\alpha, s, x} d t+F\left(t, X_{t}^{\alpha, s, x}\right) d t+G\left(t, X_{t}^{\alpha, s, x}\right) d W_{t}^{\mathcal{S}, \alpha}, \quad t \in[s, T] \\
X_{s}^{\alpha, s, x}=x
\end{array}\right.
$$

In particular (notice that the above equation enjoys strong existence, in probabilistic sense, and pathwise uniqueness) $X_{t}^{\alpha, s, x}$ turns out to be adapted to $\left(\mathcal{F}_{t}^{\mathcal{S}, \alpha}\right)_{t \geq s}$.

In $\left(\Omega^{\mathcal{S}}, \mathcal{F}^{\mathcal{S}},\left(\mathcal{F}_{t}^{\mathcal{S}, \alpha}\right)_{t \geq 0}, \mathbb{P}^{\mathcal{S}, \alpha}\right)$ we consider the solution $\left(\widetilde{Y}^{s, x}, \widetilde{Z}^{s, x}, \widetilde{K}^{s, x}\right)$ of the following reflected backward stochastic differential equation:

$$
\left\{\begin{array}{l}
-d \widetilde{Y}_{t}^{s, x}=\psi\left(s, X_{t}^{\alpha, s, x}, \widetilde{Z}_{t}^{s, x}\right) d t+d \widetilde{K}_{t}^{s, x}-\widetilde{Z}_{t}^{s, x} d W_{t}^{\mathcal{S}, \alpha}, \quad t \in[0, T], \\
\widetilde{Y}_{T}^{s, x}=\phi\left(X_{T}^{\alpha, s, x}\right), \\
\widetilde{Y}_{t}^{s, x} \geq h\left(t, X_{t}^{\alpha, s, x}\right), \\
\int_{0}^{T}\left(\widetilde{Y}_{t}^{s, x}-h\left(t, X_{t}^{\alpha, s, x}\right)\right) d \widetilde{K}_{t}^{s, x}=0 .
\end{array}\right.
$$

By its construction we know that the law of the solution to equation (2.15) does not depend on the choice of the specific probability space $(\Omega, \mathcal{E}, \mathbb{P})$ and of the Wiener process $\left(W_{t}\right)$. Therefore the law of the processes $\widetilde{Y}^{s, x}, \widetilde{Z}^{s, x}$ and $\widetilde{K}^{s, x}$ defined above do not depend neither on the admissible setting $\mathcal{S}$ nor on the admissible control $\alpha$. In particular $\widetilde{Y}_{s}^{s, x}$ is a well defind real number (regardless of the choice of $\mathcal{S}$ and $\alpha$ ).

We argue as in [6], Proposition 2.3. Rewriting (4.6) in terms of the original noise $\left(W^{\mathcal{S}}\right)$ and integrating it between $s$ and any $\left(\mathcal{F}_{s}^{\mathcal{S}}\right)$-stopping time $\tau$, we get that $\mathbb{P}$-a.s.:

$\widetilde{Y}_{s}^{s, x}=\widetilde{Y}_{\tau}^{s, x}+\int_{s}^{\tau} \psi\left(r, X_{r}^{\alpha, s, x}, \widetilde{Z}_{r}^{s, x}\right) d r+\widetilde{K}_{\tau}^{s, x}-\widetilde{K}_{t}^{s, x}-\int_{s}^{\tau} \widetilde{Z}_{r}^{s, x} d W_{r}^{\mathcal{S}}-\int_{s}^{\tau} \widetilde{Z}_{r}^{s, x} R\left(X_{r}^{\alpha, s, x}, \alpha_{r}\right) d r$

Noticing that $\left(\int_{0}^{t} \widetilde{Z}_{r}^{\alpha, s, x} d W_{r}^{\mathcal{S}}\right)_{t \geq 0}$ is a $\mathbb{P}^{\mathcal{S}}$-martingale and that $\widetilde{Y}_{r}^{\alpha, s, x} \geq h\left(r, X_{r}^{\alpha, s, x}\right)$; by computing expectation with respect to $\mathbb{P}^{\mathcal{S}}$ we get:

$$
\begin{aligned}
\widetilde{Y}_{s}^{s, x} & =\mathbb{E} \int_{s}^{\tau} \psi\left(r, X_{r}^{\alpha, s, x}, \widetilde{Z}_{r}^{\alpha, s, x}\right) d r-\mathbb{E} \int_{s}^{\tau} \widetilde{Z}_{r}^{\alpha, s, x} \alpha_{r} d r \\
& \left.+\mathbb{E}\left[\widetilde{K}_{\tau}^{s, x}-\widetilde{K}_{t}^{s, x}\right]+\mathbb{E} \widetilde{Y}_{\tau}^{s, x} \chi_{\{\tau<T\}}+\mathbb{E} \phi\left(X_{T}^{\alpha, s, x}\right) \chi_{\{\tau=T\}}\right],
\end{aligned}
$$

Finally adding and subtracting the current cost we have:

$$
\begin{aligned}
\widetilde{Y}_{s}^{s, x}= & J(s, x, \tau, \alpha)+\mathbb{E}\left[\widetilde{Y}_{s}^{s, x}-h\left(\tau, X_{\tau}^{\alpha, s, x}\right)\right]+\mathbb{E}\left[\widetilde{K}_{\tau}^{s, x}-\widetilde{K}_{s}^{s, x}\right] \\
& +\mathbb{E} \int_{s}^{\tau}\left[\psi\left(r, X_{r}^{\alpha, s, x}, \widetilde{Z}_{r}^{\alpha, s, x}\right)-l\left(r, X_{r}^{\alpha, s, x}, \alpha_{r}\right)-Z_{r}^{s, x} \alpha_{r}\right] d r .
\end{aligned}
$$

We have therefore proved the following result 
Theorem 4.1 For every admissible setting $\mathcal{S}$ and every admissible control $u \in \mathcal{U}^{\mathcal{S}}$ we have:

$$
J(s, x, \tau, \alpha) \leq \widetilde{Y}_{s}^{s, x},
$$

moreover the equality holds if and only if

$$
\begin{gathered}
\psi\left(r, X_{r}^{\alpha, s, x}, \widetilde{Z}_{r}^{s, x}\right)-l\left(r, X_{r}^{\alpha, s, x}, \alpha_{r}\right)-Z_{r}^{s, x} \alpha_{r}=0, \quad \mathbb{P}^{\mathcal{S}}-\text { a.s. for a.e. } r \in[s, \tau] \\
\widetilde{K}_{\tau}^{s, x}-\widetilde{K}_{s}^{s, x}=0, \quad \mathbb{P}^{\mathcal{S}}-\text { a.s. } \\
\widetilde{Y}_{\tau}^{s, x} I_{\{\tau<T\}}=h\left(\tau, X_{\tau}^{\alpha, s, x}\right) I_{\{\tau<T\}}, \quad \mathbb{P}^{\mathcal{S}}-\text { a.s. }
\end{gathered}
$$

Remark 4.2 Fix an admissible setting $\mathcal{S}$, an admissible control $\alpha \in \mathcal{U}^{\mathcal{S}}$ and let $\bar{\tau}$ be defined as follows:

$$
\bar{\tau}=\inf \left\{t \leq r \leq T: \widetilde{Y}_{r}^{s, x}=h\left(r, X_{r}^{\alpha, s, x}\right)\right\} \wedge T .
$$

Then condition $\int_{0}^{T}\left(\widetilde{Y}_{t}^{s, x}-h\left(t, X_{t}^{\alpha, s, x}\right)\right) d \widetilde{K}_{t}^{s, x}=0$ together with continuity and monotonicity of $\widetilde{K}$ implies that:

$$
\widetilde{K}_{\bar{\tau}}^{s, x}-\widetilde{K}_{s}^{s, x}=0 .
$$

Moreover (4.10) follows by definition. Consequently we have:

$$
\widetilde{Y}_{s}^{s, x}=J(s, x, \bar{\tau}, \alpha)+\mathbb{E} \int_{s}^{\bar{\tau}}\left[\psi\left(r, X_{r}^{\alpha, s, x}, \widetilde{Z}_{r}^{\alpha, s, x}\right)-l\left(r, X_{r}^{\alpha, s, x}, \alpha_{r}\right)-Z_{r}^{\alpha, s, x} \alpha_{r}\right] d r .
$$

Taking into account equations (4.5), (4.6) and Proposition 3.3 the above results can be refomulated as follows.

Corollary 4.3 Let $u$ be the minimal mild supersolution to the obstacle problem and let $\zeta$ be any element of its generalized gradient. Given any admissible setting $\mathcal{S}$ and any admissible control $\alpha \in \mathcal{U}^{\mathcal{S}}$ we have:

$$
J(s, x, \tau, \alpha) \leq u(s, x)
$$

moreover the equality holds if and only if the following holds $\mathbb{P}^{\mathcal{S}}$ - a.s. for a.e.r $\in[s, \tau]$

$$
\begin{gathered}
\psi\left(r, X_{r}^{\alpha, s, x}, \zeta\left(r, X_{r}^{\alpha, s, x}\right)\right)-l\left(r, X_{r}^{\alpha, s, x}, \alpha_{r}\right)-\zeta\left(r, X_{r}^{\alpha, s, x}\right) \alpha_{r}=0, \\
K_{\tau}^{s, x}-\widetilde{K}_{s}^{s, x}=0, \quad \mathbb{P}-a . s ., \\
u\left(\tau, X_{\tau}^{\alpha, s, x}\right) I_{\{\tau<T\}}=h\left(\tau, X_{\tau}^{\alpha, s, x}\right) I_{\{\tau<T\},} \quad \mathbb{P}-a . s . .
\end{gathered}
$$

Finally if

$$
\bar{\tau}=\inf \left\{t \leq r \leq T: u\left(r, X_{r}^{\alpha, s, x}\right)=h\left(r, X_{r}^{\alpha, s, x}\right)\right\} \wedge T .
$$

then the equality holds if and only if (4.13) holds.

We come now to the existence of optimal controls. We shall exploit the weak formulation of the control problem and select a suitable admissible setting $\overline{\mathcal{S}}$. We assume the following

Hypothesis 4.4 The maximum in the definition (4.4) is attained for all $t \in[s, T], x \in H$ and $z \in \Xi^{*}$ e.g. if we define

$$
\Gamma(s, x, z)=\{\alpha \in \mathcal{U}: z R(x, \alpha)+l(s, x, \alpha)=\psi(s, x, z)\}
$$

then $\Gamma(s, x, z) \neq \emptyset$ for every $s \in[0, T]$, every $x \in H$ and every $z \in \Xi^{*}$. 
Remark 4.5 By [1], see Theorems 8.2.10 and 8.2.11, under Hypothesis 4.4, $\Gamma$ always admits a measurable selection, i.e. there exists a measurable function $\gamma:[0, T] \times H \times \Xi^{*} \rightarrow U$ with $\gamma(s, x, z) \in \Gamma(s, x, z)$ for every $s \in[0, T]$, every $x \in H$ and every $z \in \Xi^{*}$.

Moreover, if $U$ is compact, then Hypothesis 4.4 always holds.

Theorem 4.6 Assume Hypothesis 4.4 and fix a measurable selection $\gamma$ of $\Gamma, s \in[0, T], x \in H$ and an element $\zeta$ of the generalized gradient of the minimal supersolution $u$ of the obstacle problem (3.1); then there exists at least an admissible setting $\overline{\mathcal{S}}$ in which the closed loop equation

$$
\left\{\begin{array}{l}
d \bar{X}_{t}=A \bar{X}_{t} d t+F\left(t, \bar{X}_{t}\right) d t+G\left(t, \bar{X}_{t}\right)\left[R\left(t, \gamma\left(t, \zeta\left(t, \bar{X}_{t}\right)\right)\right)+d W_{t}^{\mathcal{S}}\right], \quad t \in[s, T] \\
\bar{X}_{s}=x,
\end{array}\right.
$$

admits a mild solution.

Proof. We fix any admissible setting:

$$
\mathcal{S}=\left(\Omega^{\mathcal{S}}, \mathcal{F}^{\mathcal{S}},\left(\mathcal{F}_{t}^{\mathcal{S}}\right)_{t \geq 0}, \mathbb{P}^{\mathcal{S}},\left(W_{t}^{\overline{\mathcal{S}}}\right)_{t \geq 0}\right)
$$

and consider the uncontrolled forward SDE

$$
\left\{\begin{array}{l}
d X_{t}=A X_{t} d t+F\left(t, X_{t}\right) d t+G\left(t, X_{t}\right) d W_{t}^{\mathcal{S}}, \quad t \in[s, T] \\
X_{s}=x
\end{array}\right.
$$

By the Girsanov theorem, there exists a probability measure $\hat{\mathbb{P}}$ such that the process

$$
\hat{W}_{t}:=W_{t}^{\mathcal{S}}-\int_{s}^{t} R\left(X_{r}^{s, x}, \zeta\left(s, X_{r}^{s, x}\right)\right) d r \quad t \geq s
$$

is a cylindrical $\hat{\mathbb{P}}$-Wiener process in $\Xi$. We denote by $\left(\hat{\mathcal{F}}_{t}\right)_{t \geq s}$ its natural filtration, augmented in the usual way. Clearly $X$ solves

$$
\left\{\begin{array}{l}
d X_{t}=A X_{t} d t+F\left(t, X_{t}\right) d t+G\left(t, X_{t}\right)\left[R \left(X_{t}, \gamma\left(t, \zeta\left(t, X_{t}\right) d t+d \hat{W}_{t}\right], \quad t \in[s, T]\right.\right. \\
\hat{X}_{s}=x
\end{array}\right.
$$

and $\left(\Omega^{\mathcal{S}}, \mathcal{F}^{\mathcal{S}},\left(\hat{\mathcal{F}}_{t}\right)_{t \geq 0}, \hat{\mathbb{P}},\left(\hat{W}_{t}\right)_{t \geq 0}\right)$ is the desired admissible system.

We finally get the following

Theorem 4.7 Assume Hypothesis 4.4 and fix a measurable selection $\gamma$ of $\Gamma, s \in[0, T], x \in H$ and an element $\zeta$ of the generalized gradient of the minimal supersolution $u$ of the obstacle problem (3.1). Moreover let $\overline{\mathcal{S}}$ be an admissible setting in which the closed loop equation (4.16) admits a mild solution then there exists $\bar{\alpha} \in \mathcal{U}^{\overline{\mathcal{S}}}$ and an $\left(\mathcal{F}^{\overline{\mathcal{S}}}\right)$ stopping time $\bar{\tau}$ for which

$$
\tilde{Y}_{s}^{s, x}=u(s, x)=J(s, x, \bar{\tau}, \bar{\alpha}) .
$$

Proof: Just let $\bar{X}$ be the mild solution of equation (4.16)and define $\bar{\alpha}=\gamma\left(t, \zeta\left(t, \bar{X}_{t}\right)\right)$ clearly $\bar{X}_{t}=X^{\bar{\alpha}, s, x}$ and relation (4.13) holds. Thus by Corollary 4.3 it is enough to choose

$$
\bar{\tau}=\inf \left\{t \leq r \leq T: u\left(r, \bar{X}_{r}\right)=h\left(r, \bar{X}_{r}\right)\right\} \wedge T .
$$




\section{References}

[1] J.P. Aubin, H. Frankowska, Set-valued analysis, Systems \& Control: Foundations \& Applications, Vol. 2, Birkhäuser Boston Inc., Boston, MA, 1990.

[2] A. Bensoussan, Stochastic control by functional analysis methods. Studies in Mathematics and its Applications, 11. North-Holland Publishing Co., Amsterdam-New York, 1982.

[3] G. Da Prato, J. Zabczyk, Stochastic equations in infinite dimensions. Encyclopedia of Mathematics and its Applications 44, Cambridge University Press, 1992.

[4] G. Da Prato, J. Zabczyk, Second order partial differential equations in Hilbert spaces. L. ondon Mathematical Society Lecture Note Series, 293. Cambridge University Press, Cambridge, 2002.

[5] C. Dellacherie, P. A. Meyer Probability and Potential B: Theory of Martingales, NorthHolland Amsterdam (1982).

[6] N. El Karoui, C. Kapoudjian, E. Pardoux, S. Peng, M. C. Reflected solutions of backward SDE's, and related obstacle problems for PDE's. Ann. Probab. 25 (1997), no. 2, 702-737.

[7] N. El Karoui, S. Peng, M. C. Quenez, Backward stochastic differential equations in finance. Mathematical Finance 7 (1997), 1-71.

[8] I. Karatzas, S.E. Shreve, Steven, Brownian motion and stochastic calculus. Second edition. Graduate Texts in Mathematics, 113. Springer-Verlag, New York.

[9] D. Kelome, A. Swiech, Viscosity solutions of an infinite-dimensional Black-ScholesBarenblatt equation,Appl. Math. Optim., 47 (2003), 253-278.

[10] M. Fuhrman, G. Tessitore, Nonlinear Kolmogorov equations in infinite dimensional spaces: the backward stochastic differential equations approach and applications to optimal control. Ann. Probab. 30 (2002), 1397-1465.

[11] M. Fuhrman, G. Tessitore, The Bismut-Elworthy formula for backward SDEs and applications to nonlinear Kolmogorov equations and control in infinite dimensional spaces. Stoch. Stoch. Rep. 74 (2002), no. 1-2, 429-464.

[12] M. Fuhrman, G. Tessitore, Infinite horizon backward stochastic differential equations and elliptic equations in Hilbert spaces. Ann. Probab. 32 (2004), 607-660.

[13] M. Fuhrman, G. Tessitore, Generalized directional gradients, backward stochastic differential equations and mild solutions of semilinear parabolic equations. Appl. Math. Optim. 51 (2005), no. 3, 279-332.

[14] Y. Hu, G. Tessitore, BSDE on an infinite horizon and elliptic PDEs in infinite dimension. NoDEA Nonlinear Differential Equations Appl. 14 (2007), no. 5-6, 825-846.

[15] F. Masiero, Semilinear Kolmogorov equations and applications to stochastic optimal control, Appl. Math. Optim., 51 (2005), pp. 201-250.

[16] F. Masiero, Infinite horizon stochastic optimal control problems with degenerate noise and elliptic equations in Hilbert spaces. Appl. Math. Optim. 55 (2007), no. 3, 285-326. 
[17] E. Pardoux, S. Peng, Adapted solution of a backward stochastic differential equation. Systems and Control Lett. 14, 1990, 55-61.

[18] E. Pardoux, S. Peng, Backward stochastic differential equations and quasilinear parabolic partial differential equations, in: Stochastic partial differential equations and their applications, eds. B.L. Rozowskii, R.B. Sowers, 200-217, Lecture Notes in Control Inf. Sci. 176, Springer, 1992.

[19] J. Yong, X. Y. Zhou, Stochastic controls, Hamiltonian systems and HJB equations, Applications of Mathematics, Springer, New York, (1999). 